\title{
The Echinococcus canadensis (G7) genome: a key knowledge of parasitic platyhelminth human diseases
}

Lucas L. Maldonado 1*, Juliana Assis², Flávio M. Gomes Araújo², Anna C. M. Salim², Natalia Macchiaroli, Marcela Cucher ${ }^{1}$, Federico Camicia', Adolfo Fox' ${ }^{1}$, Mara Rosenzvit ${ }^{1}$, Guilherme Oliveira ${ }^{2,3}$ and Laura Kamenetzky ${ }^{1 *}$

\begin{abstract}
Background: The parasite Echinococcus canadensis (G7) (phylum Platyhelminthes, class Cestoda) is one of the causative agents of echinococcosis. Echinococcosis is a worldwide chronic zoonosis affecting humans as well as domestic and wild mammals, which has been reported as a prioritized neglected disease by the World Health Organisation. No genomic data, comparative genomic analyses or efficient therapeutic and diagnostic tools are available for this severe disease. The information presented in this study will help to understand the peculiar biological characters and to design species-specific control tools.

Results: We sequenced, assembled and annotated the 115-Mb genome of E. canadensis (G7). Comparative genomic analyses using whole genome data of three Echinococcus species not only confirmed the status of $E$. canadensis (G7) as a separate species but also demonstrated a high nucleotide sequences divergence in relation to E. granulosus (G1). The E. canadensis (G7) genome contains 11,449 genes with a core set of 881 orthologs shared among five cestode species. Comparative genomics revealed that there are more single nucleotide polymorphisms (SNPs) between E. canadensis (G7) and E. granulosus (G1) than between E. canadensis (G7) and E. multilocularis. This result was unexpected since $E$. canadensis (G7) and E. granulosus (G1) were considered to belong to the species complex E. granulosus sensu lato. We described SNPs in known drug targets and metabolism genes in the $E$. canadensis (G7) genome. Regarding gene regulation, we analysed three particular features: CpG island distribution along the three Echinococcus genomes, DNA methylation system and small RNA pathway. The results suggest the occurrence of yet unknown gene regulation mechanisms in Echinococcus.
\end{abstract}

Conclusions: This is the first work that addresses Echinococcus comparative genomics. The resources presented here will promote the study of mechanisms of parasite development as well as new tools for drug discovery. The availability of a high-quality genome assembly is critical for fully exploring the biology of a pathogenic organism. The E. canadensis (G7) genome presented in this study provides a unique opportunity to address the genetic diversity among the genus Echinococcus and its particular developmental features. At present, there is no unequivocal taxonomic classification of Echinococcus species; however, the genome-wide SNPs analysis performed here revealed the phylogenetic distance among these three Echinococcus species. Additional cestode genomes need to be sequenced to be able to resolve their phylogeny.

Keywords: Echinococcus genome, SNPs, Drug targets, Helminth parasites, Comparative genomics

\footnotetext{
* Correspondence: lucas.I.maldonado@gmail.com; Ikamenetzky@fmed.uba.ar

'IMPaM, CONICET, Facultad de Medicina, Universidad de Buenos Aires,

Ciudad Autónoma de Buenos Aires, Argentina

Full list of author information is available at the end of the article
} 


\section{Background}

Tapeworms belong to one of the three major groups of worms that parasitize humans, the other two comprises flukes (Trematoda) and round worms (Nematoda). Despite their public health importance, genome-wide data are currently available only for a few parasitic platyhelminth species including Schistosoma mansoni [1], Schistosoma japonicum [2], Schistosoma haematobium [3], Clonorchis sinensis [4], and the tapeworms Taenia solium, Hymenolepis microstoma, Echinococcus multilocularis, Echinococcus granulosus (G1) [5, 6] and Spirometra erinaceieuropaei [7]. Recently, the $50 \mathrm{Hel}-$ minth Genomes Initiative headed by the Wellcome Trust Sanger Institute provided several additional draft genomes of nematodes, cestodes and trematodes (ftp://ftp.sanger.ac.uk/pub/project/pathogens/HGI/).

Cystic hydatid disease is a zoonosis caused by Echinococcus granulosus sensu lato species complex which is associated with poverty and poor hygiene practices, particularly in livestock-raising communities [8]. It is a preventable condition that is recognized by the World Health Organisation as a "neglected" disease. It has been estimated that 1-3.6 million disability-adjusted life years are lost worldwide due to human cystic echinococcosis [9] and that up to $\$ 2$ billion are lost annually in the livestock industry [10]. E. granulosus s.l. has a complex life cycle, including intermediate hosts (domesticated or wild ungulates), where the hydatid cyst develops by asexual reproduction, and definitive hosts (domesticated or wild canids), where adult flatworms develop by sexual reproduction. Humans are accidentally infected by the ingestion of tapeworm eggs in contaminated food or water, or by direct contact with definitive hosts. Hydatid cysts develop mainly in the liver (65\%) and lungs (25\%), and less frequently in muscles, spleen, bones, kidneys, brain, eyes, heart and pancreas [11]. The rupture of an hydatid cyst and the sequelae of rupture are named secondary hydatid disease and are more important than the mass effect of hydatid cysts, mostly in the brain, where the mass effect has severe consequences.

Echinococcus granulosus s.l. was initially described as being composed of ten genotypes (G1 to G10) [12]. In recent years, mitochondrial phylogenetic analyses allowed to classify most of the genotypes as new species [13]. The new classification determines that E. granulosus $s$. $l$. is composed of five species: Echinococcus granulosus sensu stricto (G1/ G2/G3), Echinococcus equinus (G4), Echinococcus ortleppi (G5), Echinococcus canadensis (G6/G7/G8/G10) and Echinococcus felidis. All of them cause unilocular echinococcosis and are macroscopically indistinguishable at the larval stage (hydatid cysts). For many years they were considered to be the same species; nevertheless, some species show clear differences such as intermediate host infectivity, antigenic profile and infectivity to humans [12]. In human beings, E. granulosus $s$. $s$. and E. canadensis are the most prevalent species, representing $\sim 77$ and $\sim 22 \%$ of total worldwide cases reported for each species, respectively [14, 15]. At present, there is no clear link between Echinococcus genetic diversity and human infection features. Some reports suggest that human infections caused by $E$. canadensis (G7) presented smaller liver cysts than those caused by E. granulosus s. s. (G1) [16], and that E. canadensis (G6) is found more frequently in the brain [17]. In previous studies of E. canadensis isolates from South America we have demonstrated that G6 and G7 genotypes are genetically indistinguishable by most of the molecular markers employed [18-22]. Particularly, E. canadensis (G7) was shown to differ from E. granulosus s. s. (G1) in the rate of development in the definitive host [23]. Recently, we demonstrated that E. canadensis (G7) protoscoleces are unable to establish an infection in the murine model [24]. However, very little is known about the factors that determine its host specificity or developmental differences. In order to address biological differences among E. granulosus $s$. $l$. species, systematic experiments need to be performed involving all of the species. This approach is very difficult to carry out with Echinococcus species since biological material from natural infections is difficult to sample and it is not possible to obtain the complete life cycle neither in vivo nor in vitro.

Regard the disease control technics, albendazole is the only drug recommended by the World Health Organisation to treat cystic echinococcosis (http:// www.who.int/mediacentre/factsheets/fs377/en/, [25]). However, this drug has low dissolution, low absorption and several side-effects [26]. In addition, resistance and/or differential efficacy can potentially arise in some Echinococcus species; therefore new effective anti-echinococcosis drugs need to be urgently developed. Hence, knowledge of the universe of potential gene product targets is essential.

Despite its biological and public health importance, no genome-wide data have yet been produced for Echinococcus canadensis (G7). In this work we are describing the genome sequencing and annotation of E. canadensis (G7) genome. We are showing comparative genomics and genome-wide SNPs analyses performed among three Echinococcus species which revealed particular SNPs sites in known drug targets and metabolism genes. Specific cestode gene families were identified based on large-scale orthology comparisons of gene families across the phyla. In addition, a curated list of potential new drug targets is presented. Finally, phylogenetic analyses based on different approaches allowed to determine the genetic distance among Echinococcus species. These data contribute to the growing global resources that allow new treatments and to understand the biology and particular features of these parasites. 


\section{Results}

\section{The genome of Echinococcus canadensis (G7) and gene} annotation

The E. canadensis (G7) genome sequence was assembled from a combination of two Illumina libraries. Highquality genomic DNA was purified from a large unilocular cyst. Polymerase chain reaction (PCR) amplification of cytochrome oxidase 1 (COX1) followed by direct sequencing confirmed the (G7) genotype. Five de novo assembly strategies were performed and the best assembly of E. canadensis (G7) genome was chosen based on quality metrics such as N50, deep coverage, number of contigs, \%GC and the coverage of the E. multilocularis genome. The best assembly was achieved using SPAdes [27] (Fig. 1 and Additional file 1: 1.1). After removing putative non target contigs (lower than $1 \mathrm{~kb}$ ), the genome was composed of 9326 contigs whose quality assembly parameters were: N50 $74.6 \mathrm{~kb}, 55 \times$ depth coverage (Table 1). The E. canadensis (G7) genome assembly comprised $115 \mathrm{Mb}$ with $41.86 \%$ of GC content. Mapping and ordering of E. canadensis (G7) contigs on the E. multilocularis chromosomes resulted in 77.196.9\% of coverage and $89.1-93.1 \%$ of identity (Fig. 1c). The total number of contigs included a 13,719-bp contig length belonging to the mitochondrial genome, with $85 \%$ of coverage, $95 \%$ of genes and $99.9 \%$ of nucleotide identity in relation to the E. canadensis (G7) mitochondrial genome, currently deposited in the GenBank under the accession number AB235847 (Additional file 1: 1.2). The Core Eukaryotic Genes Mapping Approach (CEGMA) software [28] was used to assess the completeness of the genome. The gene space was estimated to be $>85.08 \%$ complete and the most conservative CEGMA reference gene sets were recovered up to $>95 \%$ rate in the E. canadensis (G7) genome. Protein-coding genes were predicted using the MAKER2 software [29] along with species-specific gene models of $E$. multilocularis and E. granulosus (G1) and transcriptomic data of E. canadensis (G7). A total of 11,449 gene model predictions were obtained comprising a gene density of 12.82 genes per $\mathrm{Mb}$ (Table 1). A total of 6842 proteins were functionally annotated as follows: 6205 proteins were associated with InterPro2GO terms, 493 were associated by using a cestode-specific orthology group and 144 were annotated by reciprocal BLAST against UniProt database. The remaining 4607 proteins were classified as hypothetical (Additional file 1: 1.3 and 1.4). The frequency of GO (Gene Ontology) terms obtained for the annotated proteins is presented in Additional file 2A. The two main categories found were binding (GO:0005488) and catalytic activity (GO:0003824), which is in accordance with the GO terms frequency observed in other cestode genomes [30]. In addition, a total of 4202 proteins of E. canadensis (G7) were assigned to one or more KO (KEGG (Kyoto
Encyclopedia of Genes and Genomes) Orthology) identifiers classified into 31 categories of the main 5 KEGG pathways (Additional file 3: 3.1 and 3.2). We found that $E$. canadensis (G7) had $656 \mathrm{KO}$ terms associated with metabolism, grouped in 11 pathways. We found $\sim 80 \%$ of KEGG pathways expected in Echinococcus. Pathways such as de novo synthesis of nucleotide bases, amino acids and lipids seem to have been lost, which is in accordance with previous results obtained by Zhang et al. and Tsai et al. [5, 6], and reinforces the dependence of Echinococcus on its host metabolites (Additional file 3: 3.3). Regarding non-coding genes, a total of 39 microRNA genes were identified in agreement with our previous results [31]. Also 5 18S, 3 5.8S and 2 28S rRNA genes were found in addition to 124 tRNA genes with $76.8-100 \%$ of identity to previously described tRNAs sequences (Additional file 1: 1.5, 1.6 and 1.7). A total of 21 tRNA gene clusters with up to 6 tRNA genes were identified, according to what has been reported for nematodes and mammalian genomes [32].

\section{Repetitive elements}

E. canadensis (G7) repeat sequences (E.canG7_rep), including simple repeats, interspersed repeats and satellite DNAs, were identified in the assembled genome using RepeatModeler [33] and an in-house flatworm repetitive database. We found that the repeat content of the parasite genome was $7.9 \%$ and included well-known repetitive elements, such as E. canadensis (G7) genotype TREG element described in previous studies by us $[18,19]$. To perform comparative genomic analyses, we selected a subset of sequences with more than $50 \%$ of coverage of the consensus repetitive sequences. This highly reliable set of repetitive elements was composed of 213 sequences and comprised $2.4 \%$ of the $E$. canadensis (G7) genome. Repetitive sequences were classified as DNA transposons (32.4\%), Long terminal repeat (LTR) retrotransposons (27.7\%), Long Interspersed Nuclear Elements (LINEs) (19.3\%), Short Interspersed Nuclear Elements (SINEs) (0.9\%) and unknown class (19.7\%) (Additional file 1: 1.8 and 1.9). Moreover, 13 microsatellite sequences representing 20 loci in the E. canadensis (G7) genome were identified as tandemly repeated multi-loci microsatellite EmsB, which have been used to study the genetic diversity of the genus Echinococcus [34, 35]. The identity of the repetitive elements between $E$. canadensis (G7) and E. granulosus (G1) ranged from $65.5 \%$ to $100 \%$, whereas between E. canadensis (G7) and E. multilocularis ranged from $57 \%$ to $100 \%$. The most frequent E.canG7_rep sequence identified was the E.canG7_Brep (548 loci), which had high similarity with E. granulosus (G1) EgBRep repetitive DNA element (98.93\% of identity) [36] (GenBank: $\times 67152.1$ ). This SINE sequence had less conservation in E. multilocularis $(82.86 \%$ of 

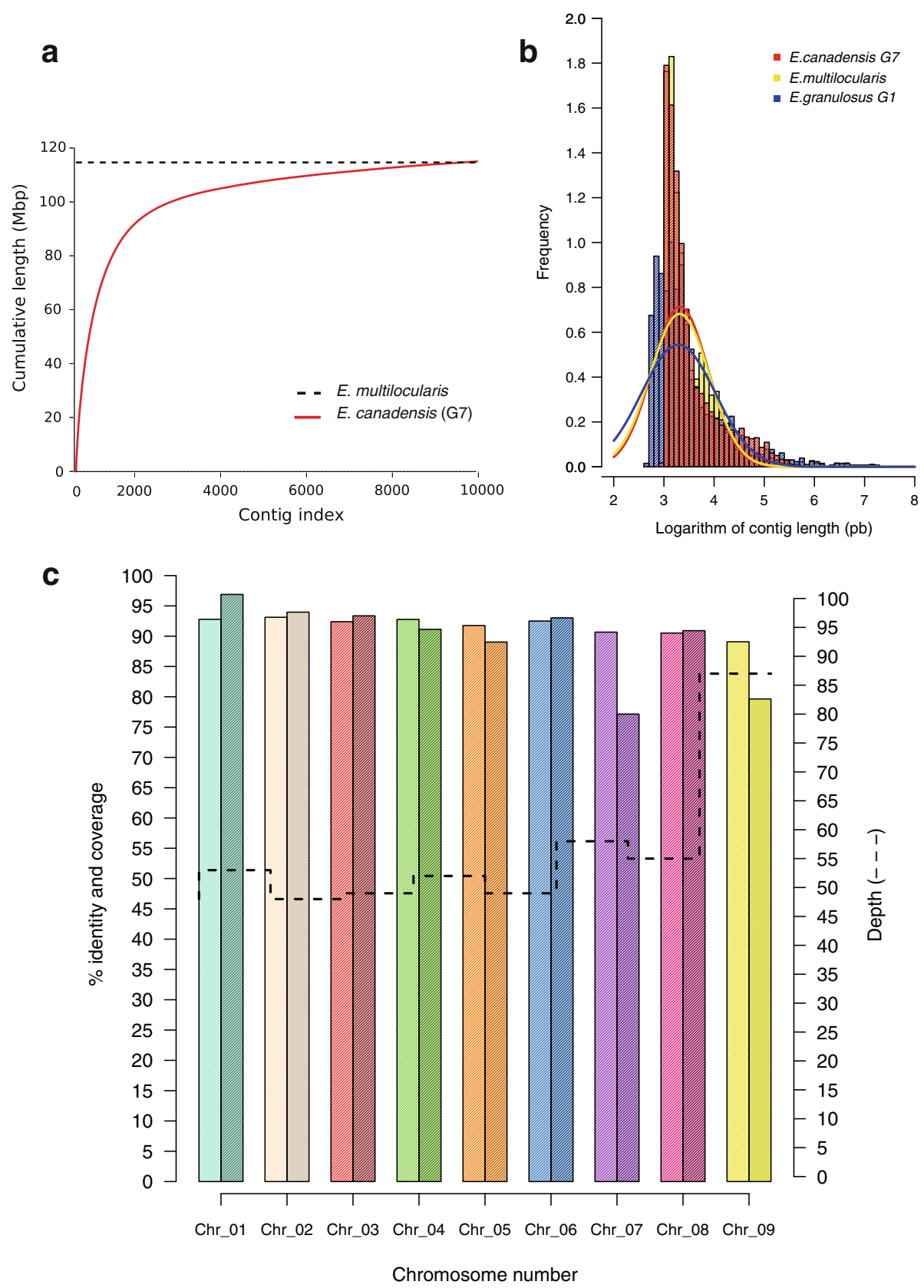

Fig. 1 Statistic measures of quality assembly. a Cumulative length distribution using E. multilocularis assembly as reference genome. $\mathbf{b}$ Echinococcus canadensis (G7) contig length distribution. The histogram represents the frequency of contigs per log contig length (bp). Lines indicate normal distribution of log contig length. c Identity, coverage (bars) and depth coverage (dashedline) of E. canadensis (G7) contigs on E. multilocularis chromosomes

identity). Also, E.canG7_Brep sequences seem to be specific for flatworms since positive hits were found in Hymenolepis microstoma, Taenia solium, Schistosoma mansoni and Schistosoma japonicum, and no sequence similarity was observed in any other organisms. The E.canG7_Brep sequence had $88.5 \%$ of identity and $70.2 \%$ of coverage in relation to terminal-repeat retrotransposon in miniature (TRIM) sequences of E. granulosus (G1)
(pathogen_EgG_scaffold_0006_Trim-1250290-1250818) found by Koziol [37]. The second most frequent repetitive element was E.canG7_rep142 (254 loci) which had a high copy number in all of the flatworm species sequenced so far, being variable in all of the Echinococcus species (90-91.3\% of identity), and included an open reading frame (ORF) encoding for pol polyprotein. Furthermore, E.canG7_rep142, E.canG7_rep39 and E.ca 
Table 1 Genome-wide statistics for the Echinococcus canadensis (G7) assembly and gene predictions

\begin{tabular}{ll}
\hline Genome statistics & \\
\hline Size of genome (Mb) & 115 \\
GC content (\%) & 42 \\
Number of contigs & 9326 \\
N50 (Kb) & 75 \\
Largest contig (Kb) & 574 \\
Deep coverage & $55 \times$ \\
Number of predicted genes & 11,449 \\
Gene density per Mb & 13 \\
Length of proteome (amino acids) & $4,915,068$ \\
Maximum protein length (amino acids) & 7886 \\
Average protein length (amino acids) & 440 \\
Average exon length (bp) & 219 \\
Median exon length (bp) & 159 \\
Average exons per transcript & 6 \\
Median exons per transcript & 4 \\
Total length of contained introns (Kb) & 40,117 \\
Average intron length (bp) & 714 \\
Median intron length (bp) & 273 \\
\hline
\end{tabular}

nG7_rep1032 contained sequences encoding for replicase domain, reverse transcriptase domain and RNA recognition domain, respectively. Recently, a novel TRIM has been found in E. multilocularis [37] but lacked evidence of active retrotransposition in E. canadensis (G7) and $E$. granulosus (G1). In addition, most of the E.canG7_rep (73\%, 156/213) detected in E. canadensis (G7) had expression evidence (RNA-seq reads) in the protoscolex and metacestode stages. E.canG7_Brep and E.canG7_rep39 had a high number of RNA-seq reads (Additional file 2B).

\section{Comparative genomics among Echinococcus species}

To determine the gene-order arrangement, we retrieved one-to-one orthologous gene pairs among E. multilocularis, E. canadensis (G7) and E. granulosus (G1) having previously ordered their contigs on E. multilocularis chromosomes and then using the OrthoMCL gene clustering (see next section). We found a high degree of conservation in the structure and organisation of genes within the three Echinococcus species (Fig. 2). The range of syntenic genes was from 89.1 to $97.1 \%$ (average $94.6 \%$ ) between E. canadensis (G7) and E. multilocularis and from 95 to $99.7 \%$ (average 98.3\%) between E. canadensis (G7) and E. granulosus (G1) (Additional file 1: 1.10). In order to perform orthology analysis, we constructed orthologs groups from a total of 14 proteomes of different organisms, including model organisms and representatives of the phylum Platyhelminthes. The total number of genes and orthology groups obtained in each organism are shown in Additional file 1: 1.11 and 1.12. From a total of 39,482 clusters of orthologous genes obtained, 6134 were present in all of the flatworm parasites and in at least one species of metazoan, among which 5203 orthologous groups were contained in all of the species of cestodes. The cestode category shared 3068 groups with at least one non-cestode species of flatworm parasites, 881 were cestode-exclusive and 560 groups were found only in Echinococcus species (Fig. 3). A total of 581 E. canadensis (G7) proteins belonging to Echinococcus specific orthologous groups were further analysed (Additional file 1: 1.13, 1.14 and 1.15 ), among which $80 \%$ were classified as hypothetical proteins. A total of 115 proteins had conserved domains and 49 proteins had molecular function GO term associated with them (Fig. 4). Two of the five GO categories grouped the $90 \%$ of all the Echinococcus specific proteins. These categories were binding (GO:0005488) and catalytic activity (GO:0003824).

\section{Expanded protein families in Echinococcus}

A total of 26 expanded protein families consisting of 10 to 66 members were found in Echinococcus (Additional file 1: 1.16). Among them, we found the heat-shock protein 70 (Hsp70) family, which has been described by Tsai et al. [5] in all of the tapeworm genomes obtained so far [6]. We also found three interesting expanded families that were present only in the cestode orthology group: GPS motif-containing protein, Ubiquitin-conjugating enzyme and Glycosyl transferase. The E. canadensis (G7) GPS motif-containing protein is related to polycystin-1, a protein involved in central signal-transduction pathways and the GPS motif (PF01825) is the responsible of protein-protein interactions. Polycystins form an expanded family of proteins composed of multiple members in fish, invertebrates, mammals and humans. Ubiquitin-conjugating enzyme is known to be involved in the ubiquitination pathway, modulating proteins degradation and protein-protein interactions. The Ubiquitin-conjugating (UBC) complex consisted of up to 19 genes in Echinococcus. Protein sequence alignments showed a high conservation of the UBC superfamily domain (PF00179) only among cestode parasites. The third expanded proteins family is the glycosyl transferases family, which is involved in glycan biosynthesis and modifications. This important pathway could play an important role in the biogenesis of the acellular carbohydrate-rich laminated layer, which is a unique Echinococcus-specific trait and one of the morphological traits that differs among Echinococcus species. These protein families are composed of 10 members that are conserved in cestodes but are very divergent in relation to other organisms (Additional file 4). 


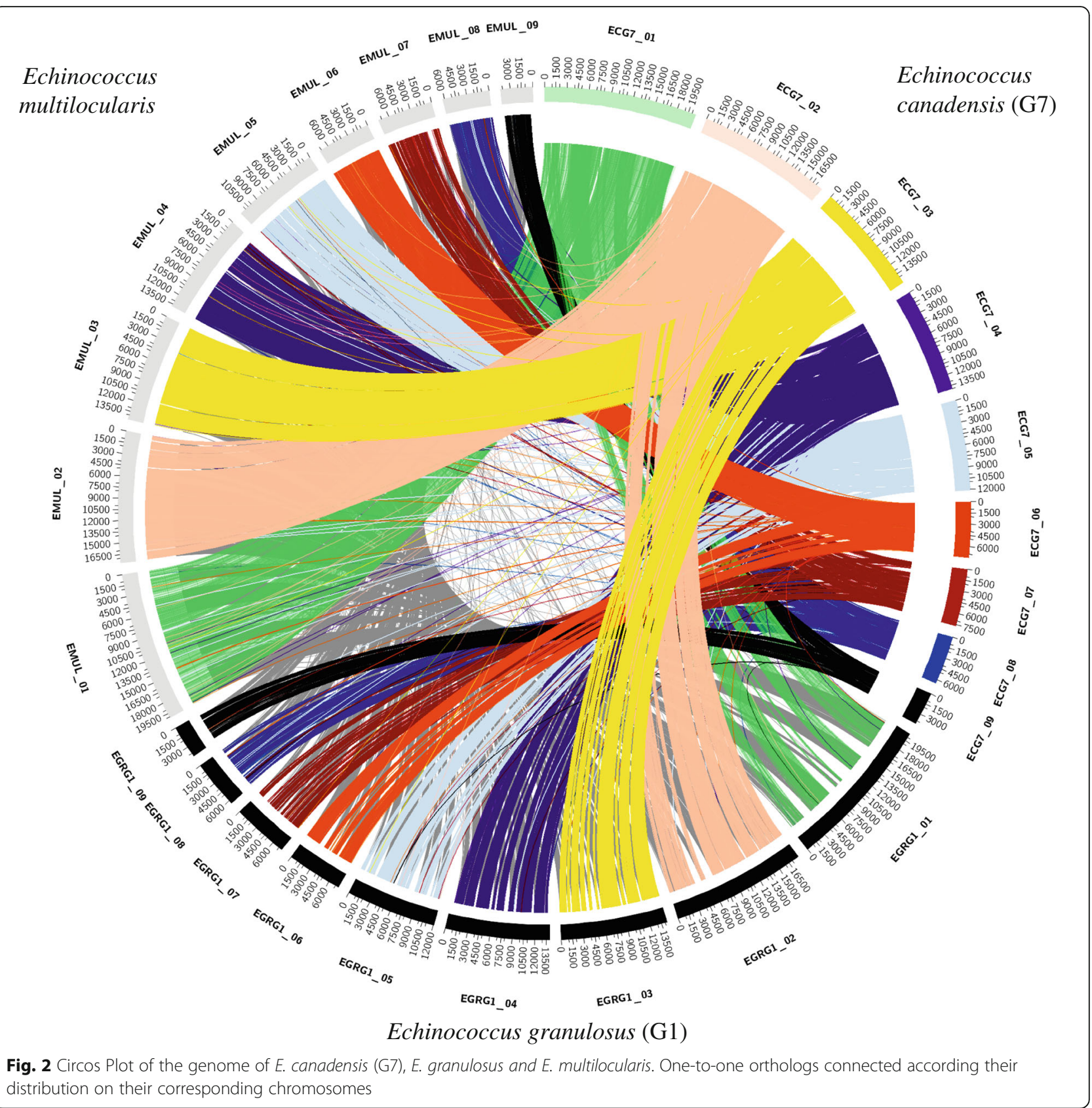

\section{Drug targets}

Protein drug targets previously described by us [6] were searched in the E. canadensis (G7) counterpart gene repertory. Comparative genomic analyses showed 89.4$100 \%$ of identity with E. granulosus and E. multilocularis proteins, whereas the range of identity with $S$. mansoni proteins was lower, from 34.9 to $97 \%$. Phylogenetic analyses including reference proteomes showed that 20 out of 21 drug targets had human orthologs (Additional file 1: 1.17). In order to obtain more specific drug target genes we selected new candidates considering the following criteria: to be present in all of the cestode species, to have high sequence conservation among cestodes (MCL score <0.8) and to be absent or to have a high degree of divergence in humans (Table 2). A total of 42 cestode proteins were selected and all these putative drug targets were grouped into 7 categories: 1 - antigens: Taeniidae antigen (AgB) and immunogenic protein Ts11; 2-defence: antimicrobial peptide; 3 - signalling: neuropeptides and peptide hormones; 4- transport: vacuolar $(\mathrm{H}+)$ ATPase $\mathrm{G}$ subunit containing protein; 5- metabolic processes: dolichol 


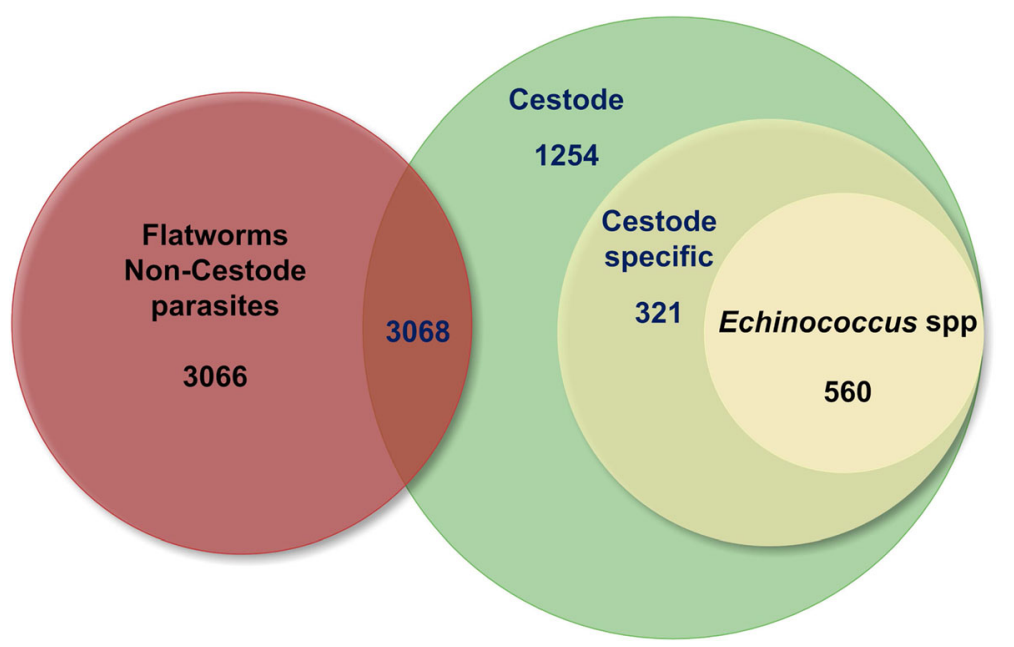

Fig. 3 Orthologous genes present exclusively in Echinococcus species. Venn diagram illustrating the number of gene clusters in each analysed group

phosphate mannosyl transferase subunit containing protein and EF-hand calcium-binding protein; 6- transcription processes: zinc finger $\mathrm{C} 2 \mathrm{H} 2$-containing protein and 7- conserved hypothetical proteins. (Additional file 1: 1.18, $1.19,1.20$ and 1.21.) These proteins were subject of manual curation and are further described in Additional file 4.

\section{Cytosine methylation in Echinococcus}

Cytosine methylation is a conserved epigenetic feature that is found throughout the phylum Platyhelminthes. Metazoan DNA methyltransferases (DNMT1, DNMT2, DNMT3a/3b) are involved in catalysing this feature by transferring a methyl group $(\mathrm{CH} 3)$ from S-adenosyl

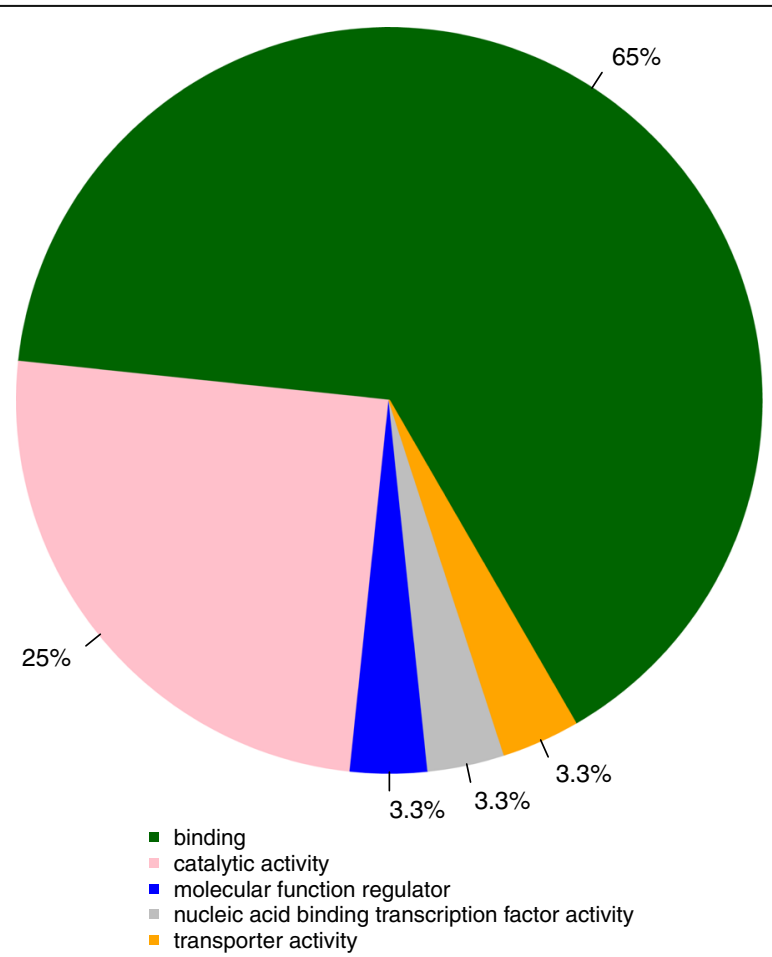

Fig. 4 Proportion of Echinococcus species-specific proteins with functional information according to different Gene Ontology (GO) categories. Gene models from E. canadensis (G7) genome were classified according to molecular function GOterms 
Table 2 E. canadensis (G7) new drug targets proteins found in cestodes but absent or highly divergent in humans

\begin{tabular}{lll}
\hline Category & Product & Ecanadensis (G7) IDs \\
\hline Antigens & Taeniidae antigen (Antigen B) & ECANG7_07838 \\
& immunogenic protein ts11 & ECANG7_01678 \\
Defense & Antimicrobial peptide tachystatin A & ECANG7_00862 \\
Sygnalling & neuropeptide-like protein & ECANG7_03703 \\
& neuropeptide spp-like & ECANG7_10139 \\
& Pancreatic hormone & ECANG7_09023 \\
Transport & Pancreatic hormone & ECANG7_05886 \\
Metabolic process & Vacuolar (H+) ATPase G subunit & ECANG7_02132 \\
Transcription processes & Dolichol phosphate mannosyltransferase subunit 3 & ECANG7_01023 \\
& EF-hand domain containing protein & ECANG7_02884 \\
\hline
\end{tabular}

OG distant less than 0.8 and present in all cestodes species analysed (stricted criteria)

methionine (SAM) to the 5-carbon (C5) position of cytosine in the genomic DNA [38]. These "epigenetic marks" are subsequently recognized as binding CpG sites by methyl-CpG-binding domain proteins (MeCP2 and MBD1-4) and converted into signals that are necessary for generating phenotypic diversity [39]. DNMTs and MBDs complexes together with other proteins constitute the core of the metazoan DNA methylation system in both vertebrate and invertebrate species. Cytosine methylation has been observed in the S. mansoni genome and this epigenetic feature was directly dependent upon the presence of enzymatically active DNMT2 [40]. DNA methylation has been described in several Platyhelminthes species and homologous DNMT2 has been found in S. mansoni [41]. DNMT2 and cytosine methylation have been identified in the $E$. multilocularis protoscoleces. DNMT2 was also identified among the gene repertory of E. canadensis (G7), but neither DNMT1 nor DNMT3 orthologs were found (Additional file 5A). MBDs have been also observed in several species of Platyhelminthes [41], which seem to be members of the ancestral MBD2/3 family [42]. Despite sharing more than $70 \%$ of amino acid identity, which is indicative of a gene duplication event, mammalian MBD2 can bind methylated cytosine within genomic DNA, whereas MBD3 cannot. Therefore, further research is needed to identify whether these new platyhelminth MBD2/3 proteins are functionally closer to methyl-CpG-binding MBD2 or to non-methyl-CpGbinding MBD3. So far, only one MBD candidate has been found in each of the platyhelminth genomes. However, we not only found the previously described MDB protein in the E. canadensis (G7) genome (EcanG7_00768), but also identified a novel MBD that showed conserved domains and characteristic motifs of the MBD domain across members of the class cestode.
The phylogenetic analysis grouped them into a specific cluster (Additional file 5B and C). These novel MBD proteins contain the $\mathrm{N}$-terminal methyl-CpG-binding domain, which consist of 73 residues, a PHD domain (PF00628) of 48 amino acids in length and a bromodomain (PF00439) toward the C-terminal comprising 86 residues.

\section{CpG islands}

CpG is the pair of adjacent nucleotides that appear in a row on the same strand of DNA linked by a phosphodiester bond. CpG islands (CGIs) are palindromic stretches of DNA comprising about 1000 base pairs long that contain a higher $\mathrm{CpG}$ density than the rest of the genome and are not methylated [43]. CpG dinucleotides in GC-rich regions can be considered gene markers and may also play an important role in the regulation of gene expression. Approximately $70 \%$ of gene promoters reside within CGIs [44]. Enhancer elements can be located between CpGs or even include CpGs, thus hiding both the core promoter qualities and the enhancer qualities of the CGI [45]. Since many transcription factors binding sites are GC-rich, CGIs are likely to enhance binding to these sites, even in the absence of common promoter elements such as TATA boxes [46]. The methylation of CGIs results in stable silencing of gene expression [47]. It was also observed that, during gametogenesis and early embryonic development, CGIs undergo differential methylation [43]. In this work, CGIs distribution was evaluated in the three Echinococcus species and particular differences were detected among distinct genomic regions. We identified 4200, 4297 and 4249 CGIs, comprising a CGI density of 36.5, 37.4 and 36.9 (\# of CGI per $\mathrm{Mb}$ ) in E. canadensis (G7), E. multilocularis and $E$. granulosus (G1), respectively. Compared to studies carried out on mammalian genomes [48], Echinococcus 

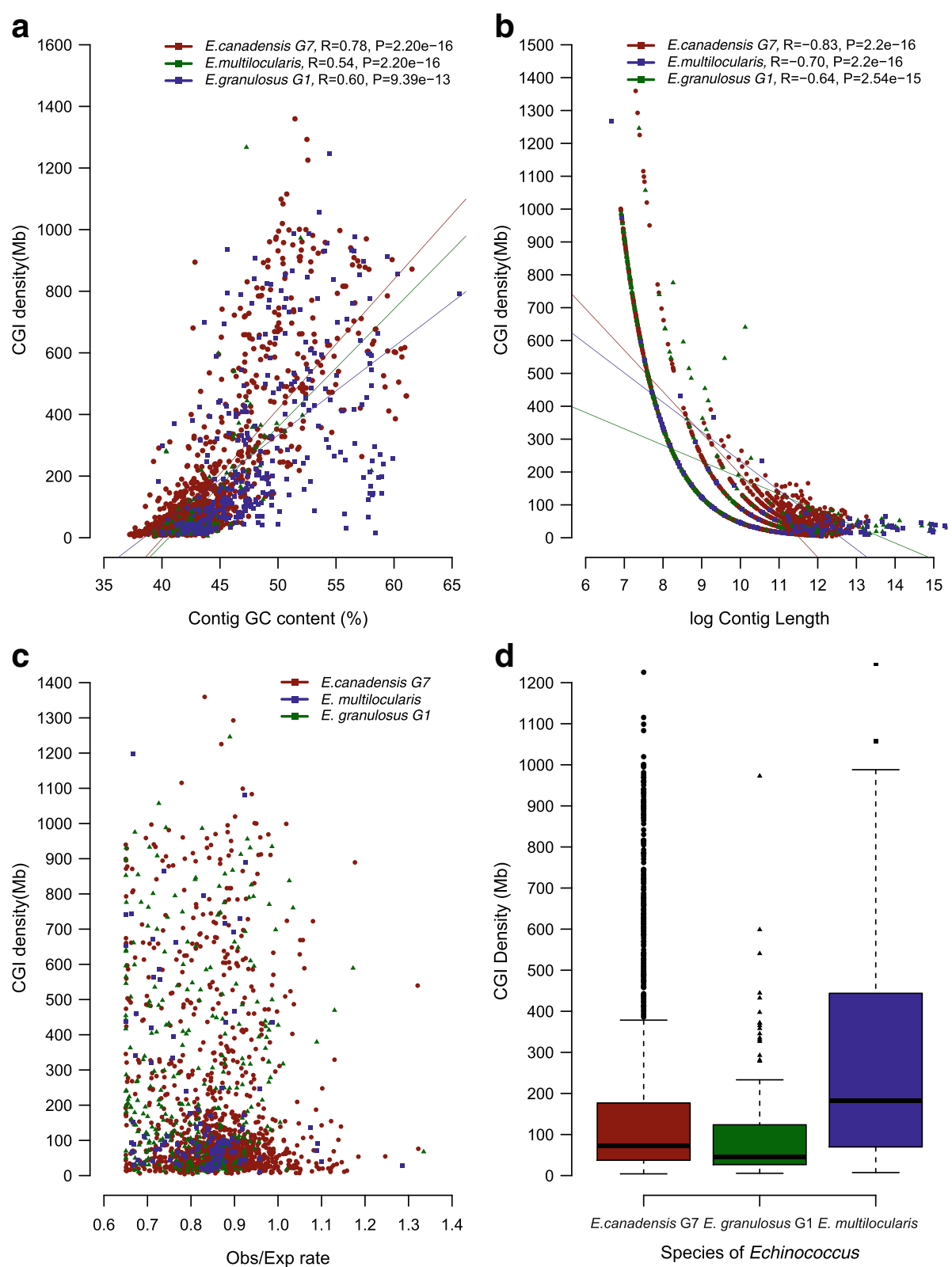

Fig. 5 Correlations between CGI density and genomic features in the genomes of the three Echinococcus species. a CGI density (per Mb) versus contig GC content (\%). b CGI density (per Mb) versus log (contig size). c CGI density (per Mb) versus contigs Obs.CpG/Exp.CpG. d CGI density (per Mb) by Echinococcus species

genomes exhibited similar length of the stretches of DNA where CGIs reside but higher levels of both, CGI density and observed/expected ratio of CpG. When we performed CGIs distribution analysis on coding regions and non-coding regions of Echinococcus genomes we observed that the number of CGIs and the CGIs density were much higher in the coding regions. We also evaluated the distribution of CGIs $5 \mathrm{~kb}$ upstream from coding regions and we identified 1363, 1637 and 1457 CGIs in E. canadensis (G7), E. multilocularis and E. granulosus (G1), respectively. The CGI density was calculated considering only those regions where CGIs were found. The values of CGI density observed were 254.9, 253.1 and 241.7 respectively and slightly lower values than in coding regions but higher than in the rest of the genome were found (Additional file 6: 6.1). Furthermore, we estimated the correlation between CGIs density and other genomic features such as genome GC content, contig size and observed/expected ratio of CpG. In this regard, we observed a significant correlation between CGIs density and genome GC content, and between CGIs density and contig size in the three genomes of 
Echinococcus (Figs. 5a and b). By contrast, there was no correlation between CGIs density and the observed/expected ratio of CpG (Fig. 5c). We also estimated CGIs density per contig length only in those contigs containing CGIs for each Echinococcus species (Fig. 5d). The median of CGIs density in E. canadensis (G7) and E. granulosus (G1) genomes was lower in relation to $E$. multilocularis genome. The correlation between CGIs density and genome GC content differed among the three analysed regions, i.e. whole genome, coding regions and upstream coding regions. The same pattern was observed for the three Echinococcus species (Additional file 7). In E. canadensis (G7), the median of distance of CGIs from the start codon of genes was roughly
$2 \mathrm{~kb}$, and the range for most of the genes was between 500 and $3500 \mathrm{bp}$ approximately. The same results were found in E. granulosus (G1) and E. multilocularis (Additional file 6: 6.2). We also identified CGIs differentially distributed along the genome of the three Echinococcus species, suggesting that the presence/absence of CGIs upstream Echinococcus genes could differentially regulate the gene expression. CGIs upstream Echinococcus genes are summarized in Additional file 6: 6.3, 6.4, 6.5, 6.6, 6.7 and 6.8. The numbers of genes differentially preceded by CGIs were 501 between E. canadensis (G7) and E. granulosus (G1), 548 between E. canadensis (G7) and E. multilocularis and 357 between $E$. multilocularis and E. granulosus (G1). Interestingly, we found drug target

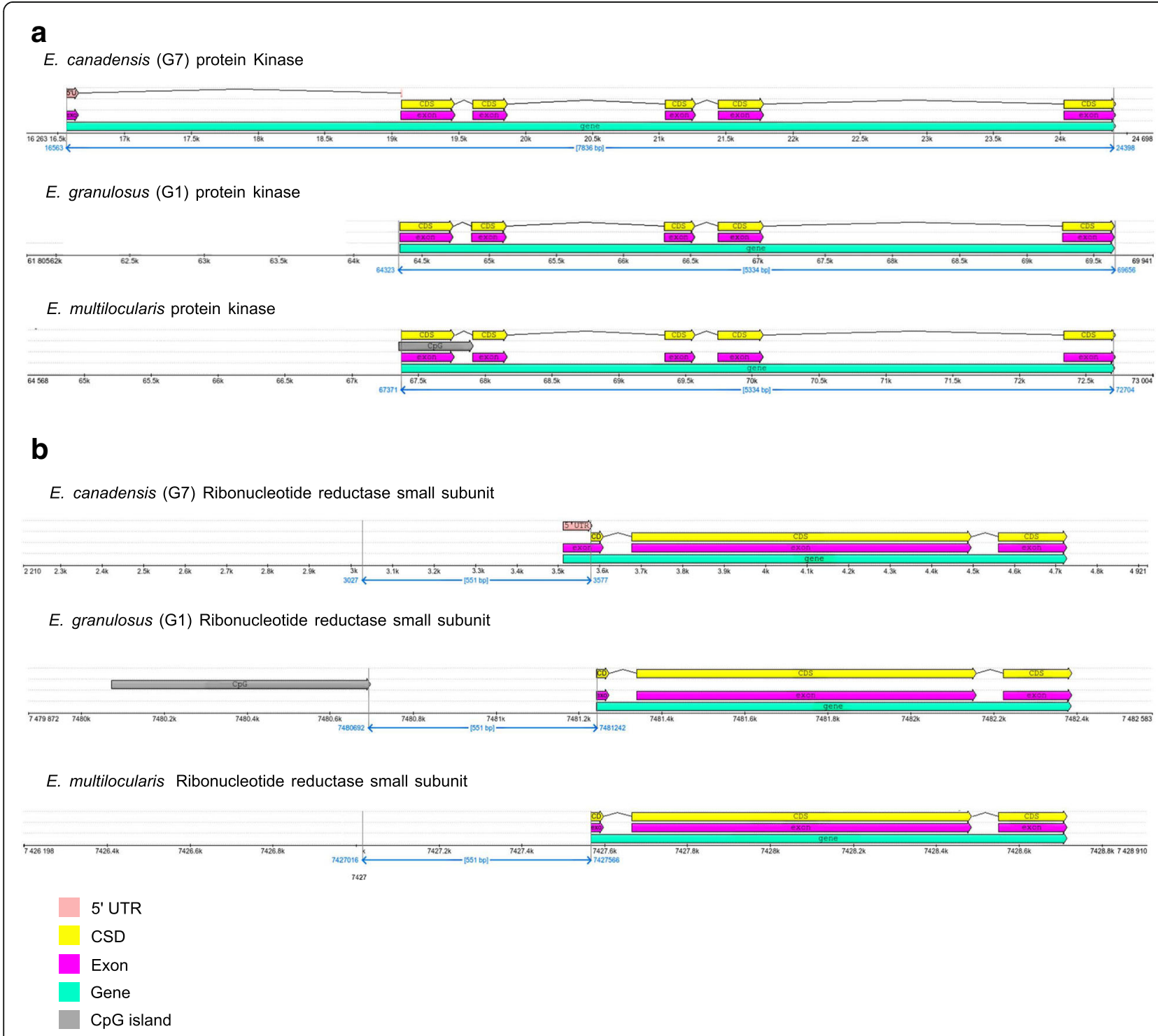

Fig. 6 Genomic context of CpG islands associated with genes in Echinococcus. a ECANG7_03687 that codes for protein kinase . b ECANG7_06911 that codes for ribonucleotide reductase small subunit. 
genes differentially preceded by CGIs. The genomic context for the ribonucleotide reductase small subunit and for the protein kinase that are differentially preceded by CGIs is shown in Fig. 6 for the three Echinococcus species.

\section{Small RNA pathway}

Since the discovery of miRNAs in cestodes [49], many reports have highlighted the importance of miRNAs in several parasite species [50] because of their relevant roles in mammals where they control several pathways, such as developmental timing, haematopoiesis, organogenesis, apoptosis, cell proliferation and tumorigenesis. An important effector in miRNA pathways is the protein Argonaute (Ago). Phylogenetic analyses showed two clades for the Ago proteins: one clade grouped a highly conserved Ago protein found in all of the organisms. The other clade grouped new Ago/Piwi-like proteins present only in flatworm parasites and are very different from any other Ago/Piwi-like protein described in humans, mice and flies [6]. In order to further characterise Ago proteins in Echinococcus (G7) we identified the OrthoMCL clusters that included the Ago proteins and searched for the characteristic PAZ and PIWI domains. We found 4 Ago proteins with an amino acid identity that ranged between 69 and $99.7 \%$. We confirmed the gene expression by RT-qPCR for the 4 Agos in E. canadensis (G7) metacestodes (Additional file 8A and B). In order to identify conserved domains and the possible interaction with a miRNA, 3D structures of Ago proteins were obtained by comparative modelling. For analysis purposes, we grouped the proteins into 4 groups (1 to 4 ), where the first one contained the conserved Ago proteins, which have high identity with the mammal Ago-2, and the three other groups contained the Echinococcus proteins of the new Ago clade (Additional file 8C). Each of the structures obtained showed RNAseH-like fold in concordance with the Ago/Piwi family proteins studied so far [51, 52] (Additional file 8D and $\mathrm{E}$ ), except for proteins from group 3 , which lack the N-terminal region and a stretch of the PAZ domain.

Ago proteins form a kind of channel or pocket in which the miRNA resides and interacts with residues of Piwi, Mid and PAZ domains and the target mRNA has access to mate with the miRNA [51]. The ArgoN domain located in $\mathrm{N}$-terminal region contributes to the final conformation and has been described as being involved in the slicer activity. In spite of overall domain conservation, some particular residues are divergent between canonical and the platyhelminthic Ago/Piwi proteins, but they are conserved among proteins of this new clade (Additional file 8F and G). Moreover, the presence of motifs L1 and L2 adjacent to the PAZ domain, which consists of two beta-sheets and two alpha-helices, respectively, confirmed that proteins from Echinococcus Ago Group 1 belonged to the mammal conserved Ago family (Additional file $8 \mathrm{H}$ ). In contrast to mammals, where other Agos lack the core related to slicer activity, we found the "DEDH" core in all of the Echinococcus Ago protein groups located in the Piwi domain which is decisive for the slicer activity of the protein on the target mRNA [52]. Furthermore, the presence of two defined motifs (Motif I and Motif II) at the N-terminal is also needed for the slicer activity (Additional file 8I and J). In humans, the methionine in Motif I plays an important role in the slicer activity of the messenger. On the other hand, Motif II comprises two alpha-helices on the exposed surface of the protein, which is involved in the activation of the RNA-induced silencing complex (RISC) and removal of the passenger strand [52]. In Echinococcus, the secondary structure of Motif I and II is conserved, but the expected methionine of the Motif I in the $\mathrm{N}$-terminal region is not present in any Ago protein. The Ago proteins of the group 2 of E. canadensis (G7) and E. granulosus (G1) lack the domain ArgoN but present the ACT_6 domain instead. ACT domains bind to amino acids and regulate associated enzyme domains that could alter the interaction with RISC. Finally, we searched for residues involved in binding the seed region of the miRNA. The amino acid residues (QSKN) constitute a motif that bind to the miRNA seed region in the Echinococcus Ago Group 1 which is conserved in relation to human Ago-2, whereas the proteins motif of the new Ago- Piwi-like clade (Groups 2 to 4 ) have a different amino acids sequence. Surprisingly, this motif is conserved among the proteins of this new clade. The seed amino acid sequence of proteins in these groups is KDGT, except for the E. multilocularis EmuJ_000911600 protein that changes an aspartic acid (D) for a glutamic acid (E) (Additional file 8K).

\section{Whole genome Echinococcus single nucleotide polymorphisms}

Next-generation sequence reads were mapped to a reference genome to identify single nucleotide polymorphisms/variations (SNPs/SNVs, hereafter referred to as SNPs). For all the analyses; the reads were first mapped against their own reference genomes and then against the genome of the corresponding analysed species. Homozygous and heterozygous variant sites were identified and were marked in both the reference and the alternative allele (see the Methods section for details). First, we evaluated the intraspecific variation in each Echinococcus genome. We observed the highest number of intraspecific variant sites in the E. granulosus (G1) genome, exhibiting a total of 74,585 SNPs which comprised a SNP rate of 0.64 SNPs each $1000 \mathrm{bp}$. The lowest number of intraspecific variant sites was observed in the 
E. multilocularis genome consisting of 1658 SNPs which exhibited a rate of 0.014 SNPs each $1000 \mathrm{bp}$. In regard to the E. canadensis (G7) genome, the number of intraspecific SNPs sites was 9449 resulting in a SNP rate of 0.09 SNPs each 1000 bp.

Genome-wide SNPs analyses performed among the three Echinococcus species revealed more SNPs between E. canadensis (G7) and E. granulosus (G1) than between E. canadensis (G7) and E. multilocularis. A total of 788,554 SNPs were identified between E. canadensis (G7) and E. granulosus (G1) genomes, comprising 1396 heterozygous and 777,710 homozygous SNPs. When we compared E. canadensis (G7) with E. multilocularis we found 327,802 SNPs sites comprising 215 heterozygous and 327,572 homozygous SNPs. In both cases the transition/transversion ratio was 2.97. Furthermore, the comparison of E. granulosus (G1) with E. multilocularis revealed 332,124 SNPs which included 6405 heterozygous and 325,691 homozygous SNPs and a transition/ transversion ratio of 2.90 (Additional file 3: 3.4). The number of SNPs located in introns and intergenic regions was also higher between $E$. canadensis and $E$. granulosus. By contrast the number of SNPs in coding regions among the three Echinococcus species was fairly similar (Additional file 9A and B). Regarding the analysis of polymorphic sites in coding regions, we demonstrated that the percentage of synonymous substitution was higher than the percentage of non-synonymous substitution in all of the analysed species. Moreover, the rate of missense SNPs between E. canadensis (G7) and E. granulosus (G1) was slightly higher than between $E$. canadensis (G7) and E. multilocularis (Additional file 9C), which involved 7153 more non-synonimous SNPs. To confirm these results, we performed the same analysis as above using the E. granulosus (G1) genome obtained by Zhang et al. [5], which comprised a different parasite isolate, sequence technology and assembly methods than the employed by Tsai et al. [6]. The results obtained were virtually the same for both E. granulosus (G1) genomes. Finally, we assessed the SNPs distribution that causes changes in amino acid residues in $5668 \mathrm{E}$. canadensis (G7) proteins and its respective one-to-one ortholog in E. granulosus (G1) and in E. multilocularis. This analysis revealed a higher distribution of amino acid changes per each 100 residues of protein between E. canadensis (G7) and E. granulosus (G1) than between E. granulosus (G1) and E. multilocularis, and even higher than between E. canadensis (G7) and E. multilocularis. This result showed differences among the species that were statistically significant according to the Anova test (Additional file 9D). We also analysed the distribution of missense SNPs in genes of Echinococcus species associated to KEGGs pathways classified in 35 pathways. These results showed again a higher SNP density by gene between E. canadensis (G7) and E. granuosus (G1) than between E. canadensis (G7) and E. multilocularis, and between E. granulosus (G1) and E. multilocularis too. SNPs distribution among KEGGs pathways is shown in figure (Additional file 3: 3.5 and 3.6 and 9E).

\section{SNPs validation}

In order to validate and verify polymorphisms detected by mapping NGS reads, we performed PCR amplification followed by direct sequencing of coding regions of some selected genes. This analysis was focused on validating missense SNPs; therefore the primers were designed based on exon sequences flanking at least one missense polymorphism. Since there are many SNPs among the different Echinococcus species, to select the best regions for primer design we detected exon regions with low variability. Selected regions were amplified by PCR of DNA extracted from 10 independent parasite isolates, three hydatid cyst of E. canadensis (G7), three hydatid cysts of E. granulosus (G1) and four metacestode isolates of E. multilocularis. Our results allowed us to characterise 48 polymorphisms (24 synonymous and 24 non-synonymous) as "high confidence" since all of the selected SNPs that were detected by NGS were also found by direct sequencing and in all of the analysed organisms (Additional file 3: 3.7).

\section{Phylogenetic studies of three Echinococcus species}

The study of the Echinococcus phylogeny was addressed using 3 different approaches: mitochondrial genome, single copy genes and genome-wide SNPs analysis.

The mitochondrial genome assembly in addition to all the available Echinococcus mitochondrial genomes were used for the phylogeny reconstruction whereby it was possible to classify and discriminate among the Echinococcus species. The mitochondrial phylogeny obtained was according to Nakao [53] (Additional file 10A).

From the total of 39,482 clusters of orthologous genes we identified 29 single-copy groups which were subsequently used to perform a phylogenetic analysis. The phylogenetic trees obtained recovered the monophyly of Chordata, Arthropoda, Nematoda and Platyhelminthes in agreement with previously published results $[6,30]$ (Additional file 10B and C).

Furthermore, to better understand the phylogenetic relationship between the taxa and to evaluate the contribution of SNPs to the genetic diversity among Echinococcus species, we selected only the homozygous SNPs and used them to perform a phylogenetic analysis. After removing heterozygous SNPs, the homozygous SNPs were concatenated and the resulting alignment was used to create a phylogenetic tree by implementing the Maximum Likelihood method. 


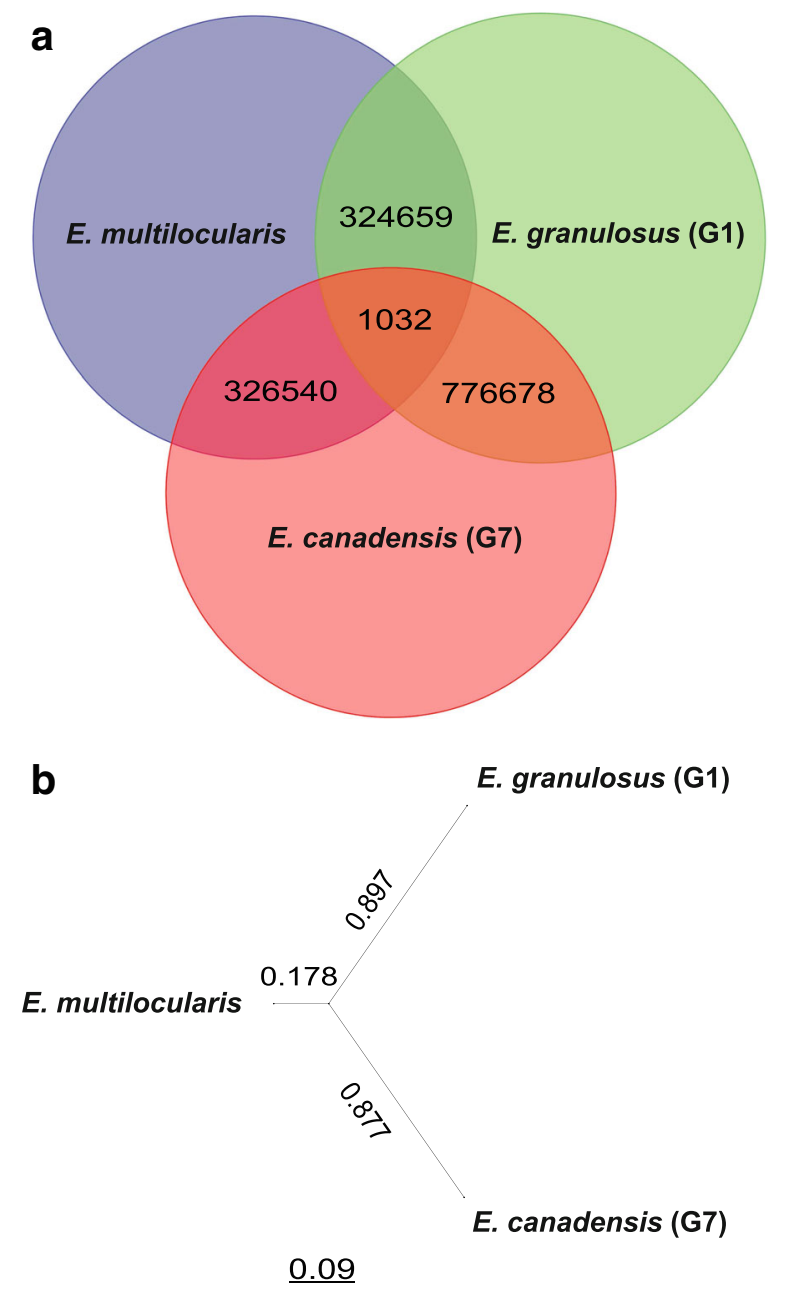

Fig. 7 SNPs and Phylogeny based on genome-wide SNPs analysis a Homozygous SNP sites between E. canadensis (G7), E. multilocularis and $E$. granulosus (G1) species. The numbers in the overlap region indicate the number of SNPs between the species. The numbers in triple overlapping indicate the number of triallelic loci. b Phylogenetic tree based on genome-wide SNPs analysis by Maximum Likelihood method

The tree topology demonstrated a higher genetic distance between E. canadensis (G7) and E. granulosus (G1) than between E. canadensis (G7) and E. multilocularis, comprising a total of 557,254 homozygous polymorphic sites. Moreover, E. canadensis (G7) and E. granulosus (G1) diverge from a common node close to E. multilocularis node (Fig. $7 \mathrm{a}$ and b). Since we observed that the number and type of changes varied according to the group of proteins analysed, random combinations of concatenated SNP sequences were also subjected to phylogenetic studies and all converged into the same topology. In addition, we registered how many polymorphic loci are shared among the Echinococcus species. For that purpose, we used a different reference genome in each one of the three rounds of mapping and we mapped the reads of the other two genomes against the selected reference. Using E. canadensis (G7) as reference, 32,142 SNPs were found at the same loci $(29,756$ had the same polymorphism). By contrast, using $E$. multilocularis as reference, we found 96,135 shared loci $(94,990$ loci with the same polymorphism). Finally, using E. granulosus (G1) as reference, 42,866 loci were found, (39,281 showed the same polymorphism) (Additional file 10D). Indeed, most of the SNPs were unique to one of the species and the highest number of shared loci was observed when the E. multilocularis genome was used as reference. It doubled and tripled the number of loci in relation to E. canadensis (G7) and E. granulosus (G1), respectively.

\section{Discussion}

The Echinococcus canadensis (G7) genome

E. canadensis (G7) is one of the most distributed parasites worldwide responsible for many human Echinococcosis cases. In recent years, several genomes of Platyhelminthes have become available; however it is yet an underexplored area. In this work we sequenced, assembled and annotated the $115-\mathrm{Mb}$ genome of $E$. canadensis (G7) whose genome size is in the same order of magnitude as other Platyhelminthes including other members of the genus Echinococcus. The E. canadensis (G7) genome contains 11,449 genes with a core set of 881 orthologs shared among five cestode species. Our work demonstrated that the genome of $E$. canadensis (G7) is highly syntenic in relation to the other Echinococcus genomes, which makes comparative genomic analysis exceptionally interesting and crucial for addressing the study of flatworms.

Comparative genomics based on SNPs analyses results were unexpected. The intra specific SNP frequency of $E$. canadensis (G7) is almost 10 fold lower than the intra specific SNP frequency found in E. granulosus (G1). A high SNPs rate had already been observed for E. granulosus (G1) by Zheng et al. [5]; even though both genomes were sequenced from genomic material from a single cyst (originated from a single egg, which was assumed to be a clone). This observation indicates a lower intra cystic genetic diversity among protoscoleces of $E$. canadensis (G7) in comparison with protoscoleces of E. granulosus (G1). The higher genetic diversity between E. canadensis (G7) and E. granulosus (G1) was indeed unexpected due to they belong to the complex $E$. granulosus sensu lato, and therefore were supposed to be phylogenetically closer to each other than to E. multilocularis. On the other hand; despite the relevant number of SNPs among the Echinococcus species, a similar distribution of SNPs for all the KEGG pathways is observed 
for every gene, suggesting that there is no bias to accumulation of SNPs in a specific pathways.

\section{New drug targets}

The analyses of protein domain sequences of new putative drug targets demonstrated that some E. canadensis (G7) proteins have greater differences with E. granulosus (G1) than with E. multilocularis. This information is relevant for developing a drug against these parasites and to be effective in geographic regions where different species coexist. As previously described by Cucher et al. [15], both species are the most frequent in human echinococcosis cases and are sympatric in many countries worldwide. At present, there are only two methods for the treatment of hydatid disease: surgery and the use of benzimidazole, but both have often unsatisfactory results [54]. Hence, novel treatment compounds are urgently needed. In this study, we identified several new potential drug targets against echinococcosis, which provided an expanded drug target repertoire. These proteins are highly conserved in cestodes but are absent or highly divergent in humans. One relevant feature would be related with the function of antimicrobial peptides. There is certain evidence that some proteins secreted by helminth parasites play a key role in modulating host immunity $[55,56]$. Furthermore, studies of Fasciola hepatica have described a secreted peptide that exhibits similar biochemical and functional characteristics to the human cathelicidin LL-37 and modulates the innate cell response by mimicking the function of host antimicrobial peptides $[57,58]$. Analysis of peptide structure in addition to orthology studies and its conservation in cestodes suggest that the E. canadensis (G7) possesses peptides that may exert an antimicrobial activity, as the proposed for the leucine-rich-repeat-(LRR)-containing proteins and the lectin-like proteins identified by Zheng et al. [5], or may play an immunomodulatory role as the proposed for the cathelicidin-like peptide of $F$. hepatica. Highly expressed secreted peptides at infectious stages of the life cycle could be involved in host-parasite crosstalk and could play an essential role in regulating host immune responses which would prolong the parasites survival in mammalian host species. Another relevant feature is related to E. canadensis (G7) neuropeptides that could be useful for performing biochemical studies of signal transduction, which have been hindered due to the inability to obtain large quantities of flatworm neuronal tissues. Indeed, these genes may represent potential drug targets for developing new therapeutic interventions or novel biopharmaceutical components.

\section{Regulation of gene expression}

A relevant genomic feature is related to the CGIs distribution that was studied in Echinococcus genomes for first time in this work. Since CGIs are considered gene markers, they are expected to have a high correlation with the gene density which indeed was found in the Echinococcus genome structure. Both the number of extant CGIs and the CGI density were much higher than in non-coding regions, which is similar to what was observed in mammalian genomes. Our studies also demonstrated that the CGI density is in correlation with the distance from the start codon of genes and the corresponding upstream region. The position CGIs and its corresponding CGIs density in upstream coding regions may help to identify promoter regions by experimental and in silico approaches; tasks that have not yet been addressed in Platyhelminthes. The diversity of eukaryotic promoters is the main obstacle for their characterisation, which is very important to understand the molecular mechanisms of gene transcription and would provide valuable information for the genetic manipulation of these parasites. Echinococcus genomes exhibited a higher CGIs density than the mammalian genomes, which is in accordance with the low levels of DNA methylation reported in some Platyhelminthes genomes and similar to many non-mammalian model organisms, such as round worms and flies, which also lack or have low levels of methylated DNA [59]. Experimental and computational studies corroborated that CGIs tend to vanish during genome evolution by a mechanism of de novo methylation of their CpG dinucleotides, which subsequently change to TpGs or CpAs due to a very high methylation-dependent transition rate [60-64]. The mammalian proteome contains many members of DNMTs and MBDs proteins, which is in accordance with the significantly lower CGIs density rate in comparison with the genomes of Echinococcus species. The high CGIs density rate $37 \mathrm{CGIs} / \mathrm{Mb}$ in the Echinococcus genomes is well explained by the absence of other members of the family of DNMTs and MBDs proteins. This phenomenon is in agreement with the low levels of methylation observed in other members of the Platyhelminthes phylum, such as $E$. multilocularis, the monogenean Protopolystoma xenopodi and the turbellarian Polycelis nigra and Macrostomum lignano species $[41,65]$. E. canadensis (G7) also provides an interesting model for studying fine-tune gene regulation during parasite development, because protoscoleces can rapidly respond to environment signals, giving rise to hydatid cyst or adult worms. On the other hand, since the E. canadensis (G7) genome has a small number of members of the DNA methylation system, as well as a novel and genus-specific MBD protein family member, this organism will provide an important parasite model for studying the evolution of methylation in Platyhelminthes.

Another regulatory mechanism is related to the differential expression of small RNAs during parasite development in E. canadensis (G7) [31]. We performed 
structural analyses of Ago proteins to gain further insight into small RNA pathways. Computational analyses including phylogenetic and protein structure studies confirm a new protein Ago clade in Echinococcus and suggest a possible interaction with miRNA. The conservation of some particular residues could play an important role in binding small RNA, in the stabilization of the mRNA target and in the slicer activity. On the other hand, specific amino acid changes in the motif that binds the seed miRNA site were also identified and are conserved through the new Ago clade. These particularities may result into a distinctive small RNA specificity and therefore a differential genes regulation that could be evaluated in future experiments. Furthermore, since Echinococcus lacks classical Piwi proteins [6] and also seems to lack piRNA molecules [31] it would be interesting to perform studies to identify molecules that interact with this new Ago clade.

\section{Expanded protein families}

We have previously identified an expansion of the Hsp70 gene family in tapeworms [6]. In this work, we identified three cestode-specific expanded gene families that could play important roles in the parasites biology: the ubiquitin-conjugating enzyme, GPCR-proteolytic site (GPS) motif-containing protein and Glycosyl transferase. The E. canadensis (G7) GPS motif-containing protein and the ubiquitin-conjugating enzyme could be involved in important pathways, such as cell signalling and protein-protein interaction. Echinococcus species exhibit an unusual high degree of developmental plasticity and gene expansions that could be explained by these phenomena. Glycosyl transferases are involved in glycan biosynthesis and modifications. These important pathways are involved in the biogenesis of the acellular carbohydrate-rich laminated layer, which is an Echinococcus-specific trait and one of the morphological traits that differentiate Echinococcus species. This expanded gene family presents sequences that are different from known glycosyl transferases. It could be interesting to determine whether the non-canonical enzymes have a metabolic role in metacestode development of each Echinococcus species.

\section{Phylogenetic relationship among Echinococcus species}

At present, there is not unequivocal taxonomic classification of Echinococcus species neither genomic analysis that reveals particularities among them. The phylogeny studies based on three different approaches allowed us to understand the phylogenetic relationship among these three species. Firstly, the reconstruction of the mitochondrial phylogeny from complete mitochondrial genomes confirms the monophyly of the E. canadensis species. Moreover; the phylogenetic tree topology of single-copy genes based on amino acid or nucleotide sequences demonstrates the status of E. canadensis (G7), E. multilocularis and E. granulosus (G1) as different species. However, slightly different topologies are observed when protein or nucleotide sequences are used for the phylogeny reconstruction, which make this analysis robust enough to discriminate among species but it is not sufficient to define a common ancestor. Furthermore, considering only coding regions, the analyses could be biased due to sampling bias and/or due to the small divergence rate among proteins, therefore the real genetic distance could be underestimated. Otherwise, wholegenome sequencing and a SNP-based approach provided the requisite level of genetic detail to resolve the paradigm of these Echinococcus species. This analysis demonstrates that E. canadensis (G7) and E. granulosus (G1) are phylogenetically more distant to each other due to a higher rate of genetic diversity and suggests that $E$. multilocularis would be the ancestral species of both. This result is in accordance with the phylogeny reconstructed from single-copy genes based on nucleotide sequences here obtained and with previous studies carried out by Saarma et al. [66]. The fact that E. canadensis (G7) and E. granulosus (G1) share more homozygous polymorphic loci with the same variant reinforces the hypothesis of a basal E. multilocularis that accumulated mutations over time until a speciation phenomenon occurred. Afterwards they would have diverged independently by increasing the genetic diversity. Indeed, whole genome sequencing is crucial not only for exposing differences among the species, but also for unequivocally defining the phylogeny and the evolutionary history of these parasites and other species. Hence, additional cestode genomes need to be sequenced in order to understand the complete evolutionary history and to obtain an accurate Echinococcus phylogeny.

\section{Conclusions}

In the current study we are presenting a new genome of Echinococcus. We sequenced, assembled and annotated the genome of the human flatworm parasite E. canadensis (G7) which highly contributes to the source of knowledge of the flatworm biology. The present work focused on the description of a group of genes that are involved in parasite development and survival, metabolic features and relevant genomic structures involved in gene expression regulation. And finally; we performed a thorough genetic variability analysis among the Echinococcus species that was taken in advantage to perform phylogenetic analyses. These results lay the groundwork for further research of Echinococcus phylogeny and many others aspects of the evolutionary history of these parasites. The resources given in this work not only 
promote the study of parasite developmental mechanisms, but will also provide new tools for drug discovery and control strategies.

\section{Methods}

Data availability

The assembled sequences of the E. canadensis (G7) genome were deposited in ENA (BioProject PRJEB8992, https://www.ebi.ac.uk/ena/data/view/PRJEB8992) and Wormbase Parasite (http://parasite.wormbase.org/Echinococcus_canadensis_prjeb8992/Info/Index/). Data on orthology groups can be downloaded from the web page of FlatDB project (http://www.bmhid.org.ar/flatdb/).

\section{Sample collection, DNA extraction and next-generation sequencing Parasites material}

All E. canadensis (G7) materials were collected from Buenos Aires, Argentina. Fertile hydatid cysts were obtained from the livers of naturally infected pigs provided by abattoirs from Buenos Aires, Argentina. The animals involved in this study were not subjected to any experimental procedure. All of the samples used in the study were collected post-mortem in commercial abattoirs. For genome sequencing purposes, we collected a large unilocular cyst from a pig's liver and the hydatid fluid was aseptically aspirated from cysts with a syringe. Protoscoleces (PS) were recovered from aspirated fluid and extensively washed in Phosphate Buffer Saline (PBS) to remove dead PS and cyst wall debris. One fraction of freshly isolated PS from each cyst was used for determining viability by eosine exclusion test. Samples showing more than $90 \%$ of viability were selected for DNA isolation. The species and genotype were determined by sequencing a fragment of the mitochondrial cytochrome c oxidase subunit 1 (CO1) [49, 67]. The resulting species and genotype was E. canadensis (G7).

\section{DNA isolation, library construction and sequencing}

PS were treated with pepsin in order to eliminate the remaining host tissue, and proteinase $\mathrm{K}$ was used to break cell wall and release the genetic material. Isolation of high-quality genomic DNA was performed by phenol/ chloroform method. Samples were quantified using a Qubit Fluorometer (Invitrogen) and quality was evaluated by rate OD 260/280 and OD 260/230 using a NanoDrop (ThermoFisher Scientific). Library preparation and miSEQ Illumina sequencing were performed at the Genomics and Computational Biology Group at Centro de Pesquisa René Rachou (CPqRR), Oswaldo Cruz Foundation, Minas Gerais, Brazil. For each library preparation, 50 ng of DNA were subjected to reaction of random tagmentation, and DNA was simultaneously fragmented and linked to specific adapters using Nextera ${ }^{\circ}$ XT DNA Sample Preparation
Kit, according to the manufacturer's instructions. Then, genomic DNA was purified and subjected to an amplification reaction using primers complementary to the adapters. The products were quantified by qPCR using $\mathrm{KAPA}^{\mathrm{mi}} \mathrm{SYBR}^{\circ}$ FAST qPCR Kit. Two libraries of $1050 \mathrm{pb}$ and $1200 \mathrm{pb}$ fragment size were prepared. Libraries were diluted in Tris- $\mathrm{HCl}$ solution + Tween $0.1 \%$, deposited in a flowchip and subjected to 500 and 600 sequencing cycles ( $2 \times 250$ bp and $2 \times 300$, respectively) using MiSeq v2 Reagent Kit. The images were processed and analysed with the manufacturer-supplied software. The quality of the Illumina reads was evaluated with FastQC v0.10.1, and the reads were trimmed and end-clipped to a phred score of 33 using Trimmomatic [68].

\section{RNA isolation and RT-PCR}

The metacestodes were kept in liquid nitrogen until they were used. The samples were centrifuged and the pellet was immediately carried at $-85{ }^{\circ} \mathrm{C}$ in TRIzol (ThermoFisher Scientific) reagent in suitable proportion. Subsequently, chloroform was added and the procedure was conducted as described by the manufacturer. Isolated RNA was treated with RQ1 DNase - Free RNase (Promega) following the manufacturer's protocol. cDNA was obtained using SuperScript III First-Strand Synthesis System for RT-PCR (Invitrogen, Life Technologies). Long PCR kit Enzyme Mix (Fermentas ThermoFisher Scientific) with proofreading activity was used for the amplification of cDNA of high molecular weight. The cDNA obtained was sequenced as previously described. In order to determine the degree of contamination with host RNA, RTPCR was performed using pig actin primers. The selection of reagents, temperatures and cycling time, primers and performing calibration curves for each Argonaute genes is shown in the Appendix. Finally, the selection of these variables was as follows: Real Time PCR Reagents brand Firepol Eva Green (Solis BioDyne) and primers purified by HPLC. RT-PCR reaction was performed in a thermal cycler Rotor Gene 60005 Plex (Corbett, Qiagen). Primers were designed for amplification of full messengers and RT-qPCR (Additional file 8L). Primers were also tested not to be paired with pig genes.

\section{PCR amplification and sequencing}

The amplification reaction for genotype verification was performed as described by Kamenetzky et al. [20]. Fragments for SNPs in vitro verification were selected by containing at least one SNP, thereby causing a missense mutation. Since there are many SNPs among the different Echinococcus species, to select the best regions for primer design, we detected exon regions with low variability. The PCR reaction was performed into a final 50 ul volume containing sample DNA (5-20 ng), $200 \mathrm{uM}$ of each dNTP (Invitrogen), $1.5 \mathrm{mM} \mathrm{MgCl}_{2}, 0.2 \mathrm{uM}$ of 
forward and reverse primers, and $2 \mathrm{U}$ of DNA taq polymerase (Additional file 3: 3.8). Thermocycling conditions started with denaturing at $95{ }^{\circ} \mathrm{C}$ for $5 \mathrm{~min}$, thermal cycling was performed for 35 cycles at $95^{\circ} \mathrm{C}$ for $45 \mathrm{~s}$, followed by $50 \mathrm{~s}$ at $57{ }^{\circ} \mathrm{C}$ and then by $72{ }^{\circ} \mathrm{C}$ for $60 \mathrm{~s}$. Reactions were finished by 5 -min incubation at $72{ }^{\circ} \mathrm{C}$. Amplification products were checked in $1.5 \%$ agarose gels stained with gelRed to verify the presence of a single amplification product. Sequencing was performed in an Applied Biosystems 3130 capillary sequencer using a Big-Dye terminator cycle sequencing kit, according to the manufacturer's instructions. Gene fragments were PCR-amplified from 3 representative isolates of $E$. canadensis (G7), 3 of $E$. granulosus (G1) and 4 of E. multilocularis, and they were sequenced in both strands. Base calling of chromatograms, assembly of sequences, detection of polymorphisms and manual inspection of assembled sequences and polymorphisms was done using a software package composed of Phred - (version phred-dist-071220.c), Phrap (version 1.090518) and Consed (version 0.29). Basecalling of chromatograms was done by Phred. Sequences were then assembled by Phrap-Consed. All candidate SNPs were identified and subjected to manual inspection.

\section{De novo assembly of E. canadensis (G7) NGS reads and removal of non-target sequences}

The E. canadensis (G7) genome sequence was assembled from a combination of the two libraries sequenced using the Illumina MiSeq platform. Sequence data were screened against host genome. In order to optimally assemble the genome of E. canadensis (G7), we tested different de novo assemblers and measured statistical parameters to define the best assembly. Velvet1.2.07 [69], SOAPdenovo2 [70] and SPAdes 3.6 [27, 71] were used on the Illumina reads. Reads and obtained contigs were reused to generate the high-quality genomes using PAGIT IMAGE [72]; ICORN [73]; ABACAS [74]. This initial assembly process resulted in the assembled genome version 1. Metrics of the quality of the assemblies obtained by DBG approaches were evaluated by QUAST [75] based on standard assembly metrics, such as N50, total number of contigs and total length of the assembly. In addition, the conjunction of all the information was employed to select the best assembly (Additional file 1: 1.1). Putative non-target contigs and contigs that were shorter than $1 \mathrm{~kb}$ in length were removed before performing further analyses. The completeness of the gene space was validated using CEGMA 2.4 [28]. Genome-wide quality comparison of Echinococcus species was performed by mapping contigs against the E. multilocularis genome using ABACAS.1.3.2 [74]. Coverage and depth coverage were calculated with custom scripts. Depth coverage refers to the number of times that the same region or position in the reference genome is represented by the assembled genome. Coverage refers to the percentage of the total length of the reference genome that is represented by the assembled genome.

\section{Gene prediction and annotation}

E. canadensis (G7) specific repeat families, including simple repeats, interspersed repeats and satellite DNAs, were identified from the assembled genome using RepeatModeler [33]. Subsequently, the automated annotation pipeline MAKER 2.31 [29] was used for ab initio gene finding. For gene model predictions, a reliable group of genes and proteins of related species of the genus Echinococcus was selected from different databases in order to generate a reliable training dataset for Augustus [76] and SNAP [77]. Protein data were also incorporated through exonerate version 2.2.0 [78]. Moreover, RNA-seq data generated by the Wellcome Trust Sanger Institute was incorporated. RNA-Seq reads were mapped to the $E$. canadensis (G7) genome with Tophat v2.0.12 [79, 80] and the output was used to construct a Hidden Markov Model for GeneMark [81] through the automatic training of the eukaryotic ab initio gene-finding algorithm GeneMark-ET [82]. The automated annotation pipeline MAKER 2.31 [29] was used for structural annotation as follows: repetitive DNA was masked using RepeatMasker [83] and the E. canadensis (G7) specific repeat library initially created using Repeat Modeler [33, 83]. Gene models identified by CEGMA 2.3 [28] were passed through MAKER 2.31 [29]. Three rounds of MAKER2 were performed. A first pass of MAKER2 was run using a custom protein database as physical evidence and employing GeneMark [81], SNAP [77] and Augustus [76] for ab initio predictions. Subsequently, a second pass of MAKER2 was performed using physical EST evidence from RNA-Seq from GFF3 file obtained with GeneMark-ET [82] and with all of the ab initio predictors turned off. The final pass of MAKER2 was run using physical EST evidence from RNA-Seq from GFF3 file obtained with GeneMark-ET and with all of the gene predictors turned on, plus gene models obtained in round 2 passed through MAKER2 as EST with a threshold AED score of 0.5. Thus MAKER2 then reconciled homology-based physical evidence with the results of purely ab initio predicted gene models. Transfer RNAs were searched by tRNAscan-SE 1.23 [84], each tRNA locus was confirmed by RNA central database (http://rnacentral.org/) analysis. Two adjacent tRNA genes were defined as clustered if their distance was less than 1000 nucleotides, according to Bermudez et al. [32]. Ribosomal RNAs were predicted by RNAmmer-1.2 software (www.cbs.dtu.dk/services/RNA $\mathrm{mmer} /$ ) and BLAST searches against NCBI and 5S 
rRNA (http://combio.pl/rrna/) databases. Specific miRNAs of Echinococcus were identified as previously described [31]. BLAST [85] was used to map miRNAs against the genome The data were added to final coordinates GFF file. Custom scripts were used to achieve the correct format files.

\section{Repetitive elements annotation}

Repetitive elements of E. canadensis (G7) were automatically identified using RepeatModeler v1.0.8. In addition, a reliable set of repetitive elements described for flatworms was used in homology searching against the genome. Custom scripts were used to select the best match from overlapping matches in RepeatModeler and homology searches. For comparative genomic analyses, we selected sequences with more than $50 \%$ of coverage of consensus repetitive sequences and classified them with TEclass software (a tool for automated classification of unknown eukaryotic transposable elements [86]). Sequences from RepeatModeler and from reported Echinococcus species entries were remapped on the E. canadensis (G7) genome. Only hits with e-values lower than 0.0001 and with more than $50 \%$ of coverage were counted as genome matches, and the genome localization was obtained with exonerate version 2.2.0 [78].

\section{Gene model analyses and gene annotation}

The performance of gene annotation and basic statistics for E. canadensis (G7) gene models, including average intron/exon lengths and number of introns were calculated using Eval [87]. Gene annotation was performed by a combination of three methods. All gene models were screened for known domains using InterProScan-5.748.0 [88] and InterPro2GO databases were used to assign Gene Ontology terms [89]. Gene models were subjected to a BLAST search [85] against UniprotDB and the Parasites orthology group built with OrhtoMCL v2.0.9 $[90,91]$ was used to define the final annotations.

\section{Orthologous gene groups}

The protein complements of 14 metazoan taxa (Additional file 1: 1.11) were searched for reciprocal best hits using BLAST [85], and the results were subsequently employed to identify orthologous gene clusters by using the MCL algorithm [91]. An inflation value was set in 1.5 and a subset of proteins was clustered according to taxa and other criteria summarized in Additional file 1: 1.12. To detect expanded protein families, orthology groups built with OrthoMCL were used. Clusters containing more than 3 proteins per species were identified in all of the protozoa species and in Echinococcus phyla.

\section{Drug targets}

In order to identify cestode-specific genes that are absent in humans, we selected orthologous genes of $E$. canadensis (G7) from orthology groups. A stringent inflation value of 0.8 was used to perform clustering of proteins that had already been reported as drug targets. In order to obtain more specific drug target genes we selected new candidates considering the following criteria: to be present in all of the cestode species, to have high sequence conservation among cestodes (MCL score $<0.8)$ and to be absent or to have a high degree of divergence in humans (Table 2). Phylogenetic analyses were performed for all of the orthology groups selected by ClustalX multiple alignments and maximum likelihood algorithm was implemented in MEGA 5 software.

\section{Domain annotation and structural analysis}

Proteins studied in this work were searched using BLAST [85] against UniProtKB/Swiss-Prot databases. Protein domains were screened against PFAM, and Prosite databases using PFAM_scan ([92] or HMMscan 3.0. Signal peptide analyses were performed using Phobius (http://www.ebi.ac.uk/Tools/pfa/phobius/) and SignalP 4.0 (http://www.cbs.dtu.dk/services/SignalP/). Secondary structure analysis was assessed with PSIPRED (http://bio inf.cs.ucl.ac.uk/psipred/) and Jpred 4 (http://www.comp bio.dundee.ac.uk/jpred/). Protein structure modelling was obtained with PHYRE2 [93] and SWISS-MODEL [94-97]. PDB databases were used for homology searching and Human Argonaute-2 - miR-20a complex (http://www.rcsb.org/pdb, [51]) was used for structural comparison analyses.

\section{CpG islands}

In order to identify $\mathrm{CpG}$ island, CgiHunterLight software tool v1.0 (http://cgihunter.bioinf.mpi-inf.mpg.de/) was applied, and Takai and Jones algorithm [98] was used, which suggests an optimal set of parameters (GC content $\geq 55 \%$, Obs CpG/Exp CpG $\geq 0.65$ and length $\geq 500 \mathrm{bp}$ ). This algorithm can effectively exclude false positive CGIs from repeats. We evaluated CpG islands in different genomic regions of the three Echinococcus species, genomic, coding and upstream coding regions using custom scripts.

\section{Genome comparison}

Genome-wide quality comparison of Echinococcus species was performed by mapping contigs against the $E$. multilocularis genome using ABACAS.1.3.2 [74], and coverage and depth coverage were calculated with custom scripts. The results of orthology analyses were used to define gene orthology regions and synteny in the chromosome context. Synteny of orthologous genes was represented by CIRCOS plot [99]. 


\section{SNPs}

All of the Illumina reads from E. canadensis (G7), E. granulosus (G1) and E. multilocularis libraries were filtered by quality and then mapped onto the Echinococcus species genomes using bowtie2 [100]. Mapping statistics were calculated with Bamtools (https://github.com/pez master31/bamtools), and duplications were marked and discarded using picard-tools v-1.129 (http://broadinstitu te.github.io/picard/). SNPs were called using Samtools [101], with the following parameters: variation frequency was set $>40 \%$ with at least 20 reads covering SNP sites, and the base quality of both reference site and variation site was $>30$. Insertion and deletions (indels) were filtered out with VCFtools [102], and SNPs with less than $10 \mathrm{pb}$ from indels were removed to avoid false positives in SNP calling. For all SNPs analyses the reads were first mapped against their own reference genomes and then against the genome of the corresponding analysed species. Heterozygous sites were only retained if both forward and reverse reads mapped against the reference and alternative allele at a given nucleotide position with more than 20 reads supporting that position. Homozygous polymorphic sites were annotated if the forward and reverse reads mapped onto the alternative allele with more than 20 reads supporting that position and if there were no reads supporting the reference allele. Homozygous and heterozygous SNPs sites were registered for all of the species. Transition/transversion ratios were calculated using VCFtools [102], and the annotation and classification of SNPs based on the effect on coding regions were carried out with SnpEff v4.0 [103]. Comparison among three of Echinococcus species was performed with custom scripts. Statistical analyses were performed by ANOVA test, with a confidence level of 95\% using R software and package car. Graphics were built using $\mathrm{R}$ software (https://www.r-project.org/).

\section{KEGGs pathways}

Metabolic pathways in the whole proteome were obtained from the KEGG database using the KAAS tool [104]. In order to provide an overview of secondary metabolite biosynthesis and a hand-picked selection of important regulatory pathways and other functional modules, we used KEGG Mapper Reconstruct Pathway for the visualization and analysis of cellular pathways.

\section{Phylogeny reconstruction with mitochondrial genomes}

Mitochondrial contigs were identified by BLAST [85] against a customized mitochondrial database of Echinococcus species. Contigs were reassembled into complete mitochondrial genome and gene annotation was performed using RATT [105]. Nucleotide sequences of 13 protein-coding genes from mitochondrial Echinococcus genomes were translated to amino acid sequences using the flatworm mitochondrial genetic code [106]. The deduced amino acid sequences were aligned by ClustalX multiple sequence aligner [107]. Phylogeny reconstruction with mitochondrial genomes was implemented using the Maximum Likelihood method based on the JTT matrix-based model performed with MEGA5 software. The bootstrap consensus tree inferred from 100 replicates was taken to represent the evolutionary history of the taxa analysed. Branches corresponding to partitions reproduced in less than $50 \%$ bootstrap were collapsed. The analysis involved 13 mitochondrial genomes with 12 proteins each (3276 amino acid sites positions). The values of each node are ML bootstrap percentages.

\section{Global phylogeny of Echinococcus and model species} Single-copy ortholog groups were selected and protein sequences were concatenated by organism species. Protein sequences were aligned using ClustalX multiple sequence aligner. Global phylogeny of Echinococcus and model species were analysed using the Maximum Likelihood method based on the JTT matrix-based model conducted in MEGA5. All of the positions with less than $60 \%$ site coverage were eliminated. The analysis involved 14 amino acid sequences from 29 single-copy genes from orthology groups. There were a total of 7001 positions in the final dataset. Nucleotide sequences from single-copy genes were aligned with transAlign software (EMBOSS), and phylogenetic analysis was performed by Maximum Likelihood method based on the Tamura-Nei model. All of the positions containing gaps and missing data were eliminated. There were a total of 14,364 positions in the final dataset. Genome-wide SNPs were used to perform phylogeny analysis as follows: only the homozygous SNPs were selected to correct for complete lineage sorting. After removing heterozygous SNPs, the homozygous SNPs loci were concatenated and the resulting alignment was used to create a phylogenetic tree by implementing the Maximum Likelihood method.

\section{Additional files}

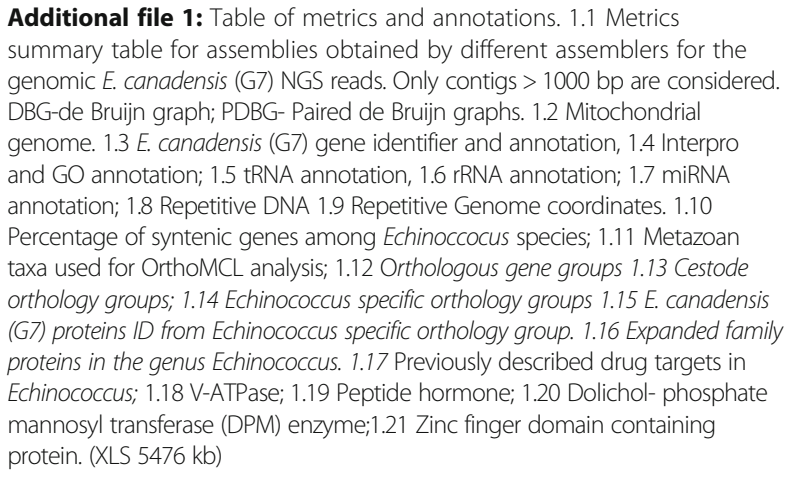

Additional file 1: Table of metrics and annotations. 1.1 Metrics summary table for assemblies obtained by different assemblers for the genomic E. canadensis (G7) NGS reads. Only contigs > 1000 bp are considered. DBG-de Bruijn graph; PDBG-Paired de Bruijn graphs. 1.2 Mitochondrial genome. 1.3 E. canadensis (G7) gene identifier and annotation, 1.4 Interpro and GO annotation; 1.5 tRNA annotation, 1.6 rRNA annotation; 1.7 miRNA annotation; 1.8 Repetitive DNA 1.9 Repetitive Genome coordinates. 1.10 Percentage of syntenic genes among Echinoccocus species; 1.11 Metazoan taxa used for OrthoMCL analysis; 1.12 Orthologous gene groups 1.13 Cestode orthology groups; 1.14 Echinococcus specific orthology groups 1.15 E. canadensis (G7) proteins ID from Echinococcus specific orthology group. 1.16 Expanded family proteins in the genus Echinococcus. 1.17 Previously described drug targets in Echinococcus; 1.18 V-ATPase; 1.19 Peptide hormone; 1.20 Dolichol- phosphate mannosyl transferase (DPM) enzyme;1.21 Zinc finger domain containing protein. (XLS $5476 \mathrm{~kb})$ 
Additional file 2: Echinococcus canadensis (G7) functional annotation and highest repetitive elements read counts. (A) Molecular function GO terms associated frequency of E. canadensis (G7). (B) E.canG7_Brep and (C) E.canG7_rep39 per-base coverage with reads from the forward strand (blue) and reads from the reverse strand (red) are shown. (PDF 295 kb)

Additional file 3: Table of metabolic pathways and associated SNPs. 3.1 E. canadensis (G7) KO; 3.2 E. canadensis pathways; 3.3 Complete pathways modules; 3.4 Number of Homozygous and heterozygous variant sites of E. canadensis (G7), E. multilocularis and E. granulosus (G1).3.5 Gene distribution in KEGG pathways; 3.6 SNPs density in KEGG pathways. 3.7 List of genes subject of experimental validation in SNPs analyses; 3.8 Table of primers for SNP validation. (XLSX $699 \mathrm{~kb}$ )

Additional file 4: Expanded proteins families and drug targets: Multiple alignment of expanded proteins families and drug target sequences of $E$. canadensis (G7) and their orthologs. (PDF $999 \mathrm{~kb}$ )

Additional file 5: DNMT and MBD proteins of Echinococcus. (A) Phylogenetic tree of Echinococcus DNMT proteins and classification in family class. (B) Phylogenetic tree of MBD proteins. New MBD protein and families classification. (C) Amino acidic residues involved in MBD motif are shown as logo plots. (PDF $295 \mathrm{~kb}$ )

Additional file 6: Table of CGls. 6.1 CpG islands and other genomic features in Echinococcus species genomes. 6.2 Summary of correlations between CGI density and genome features in the three Echinococcus species; 6.3-6.8 Differential CGls distribution upstream Echinococcus genes (presence/absent in Echinococcus genomes). (XLS 204 kb)

Additional file 7: Correlations between CGI density and coding regions in the genomes of the three Echinococcus species: (A) CGl density (per $\mathrm{Mb}$ ) versus contig $\mathrm{GC}$ content (\%). (B) CGI density (per Mb) versus log (contig size). (C) CGI density (per Mb) versus contigs Obs.CpG/Exp.CpG. (D) CGI density (per Mb) by Echinococcus species. Correlations between CGI density and upstream coding regions in the genomes of the three Echinococcus species: (E) CGI density (per Mb) versus contig GC content (\%). (F) CGl density (per Mb) versus log (contig size). (G) CGl density (per $\mathrm{Mb}$ ) versus contigs Obs.CpG/Exp.CpG. (H) CGl density (per Mb) by Echinococcus species. (I) Boxplots of average CGls distance from the start codon of genes in the three Echinococcus species. (PDF $257 \mathrm{~kb}$ )

Additional file 8: Argonaute proteins in Echinococcus. (A) Relative expression of Ago proteins in E. canadensis (G7). (B) Relative expression of Ago proteins in E. multilocularis. Actin gene Emul_190400 was used as reference. (C) Table of Echinococcus Ago proteins domains. (D) Cartoon representation of RNAse-like fold coloured by domains of Group 1 Echinococcus Ago proteins. (E) Molecular surface representation of Group 1 of Echinococcus Ago and pocket binding miRNA. (F) Overview of conserved Group 1 structures and their domain architecture. (G) Relevant conserved and non-conserved amino acid residues of Echinococcus Ago proteins. $(\mathrm{H})$ Cartoon representation of proteins from Group 1 of Echinococcus Ago: relevant and conserved residues involved in seed binding, mRNA and slicer activity are zoomed. (I) Cartoon representation of proteins from Group 2 of Echinococcus Ago: relevant and conserved residues involved in seed binding, mRNA and slicer activity are zoomed. (J) Cartoon representation of proteins from Group 4 of Echinococcus Ago: relevant and conserved residues involved in seed binding, mRNA and slicer activity are zoomed. (K) Sequence alignment of amino acid residues involved in seed binding region of the four groups of Echinococcus. (L) Table of sequence primers used for RT-qPCR. (PDF 304 kb)

Additional file 9: Single nucleotide polymorphisms in Echinococcus. (A) Number and localization of SNPs in genomic regions defined on the basis of gene architecture between the three Echinococcus species. (B) Type of substitution caused by SNPs in coding regions between the three Echinococcus species. (C) Distribution of missense mutation per each 100 residues of amino acid. (D) Statistical analyses of missense substitution each 100 amino acid residues among the pairs of Echinococcus species. The significance of the differences observed in missense SNPS was evaluated using the anova test with a confidence level of 95\%. P-values belong to Anova test significance. (E) SNPs distribution in KEGGs pathways. Measure of SNP density by calculated as the number of SNPS divided the number of genes associated with the 5 main pathways. (i) Cellular processes. (ii) Environmental Information
Processing. (iii) Organismal Systems. (iv) Metabolism. (v) Genetic Information Processing. (PDF $2885 \mathrm{~kb}$ )

Additional file 10: Global phylogeny of Echinococcus and model species. (A) Phylogeny reconstruction from complete Echinococcus mitochondrial genomes. Nucleotide sequences of 12 protein-coding genes were aligned according to Nakao et al. [53]. Phylogenetic analysis was performed using the Maximum Likelihood method based on the JTT matrix-based model. Bootstrap consensus tree was inferred from 100 replicates. Branches corresponding to partitions reproduced in less than $50 \%$ bootstrap replicates are collapsed. Mitochondrial genomes used for Echinococcus species phylogeny reconstruction: E. oligarthrus, NC_009461, Nakao et al., [108]; E. vogeli, NC_009462, Nakao et al., [108]; E. equinus, AB786665, Nakao et al., [53]; E. granulosus, AB786664, Nakao et al., [53]; E. felidis, AB732958, Nakao et al., [53]; E. multilocularis, NC_000928, Nakao et al., [109]; E. shiquicus, NC_009460, Nakao et al., [108]; E. ortleppi, NC_011122, Nakao et al., [108]; E. canadensis (G6), NC_011121, Nakao et al., [108]; E. canadensis (G7), AB235847, Nakao et al., [108]; E. canadensis (G7), PRJEB8992, this work; E. canadensis (G8), AB235848, Nakao et al., [108]; E. canadensis (G10), AB745463, Nakao et al., [53]. (B) Proteins encoded by single-copygenes were analysed using the Maximum Likelihood method based on the JTT matrix-based model. The analysis involved 14 amino acid sequences from 29 single-copy genes. There were a total of 7001 positions in the final dataset. (C) Single-copy coding DNA sequences (CDS). The analysis involved 14 nucleotide sequences from 29 single-copy genes using the Maximum Likelihood method based on the Tamura-Nei model. There were a total of 14,364 positions in the final dataset. All of the positions with less than $60 \%$ site coverage were eliminated. Evolutionary analyses were conducted in MEGA5. Hsap: Homo sapiens; Mmus: Mus musculus; Drer: Danio rerio; Bflo: Branchiostoma floridae; Dmel: Drosophila melanogaster; Cele: Caenorhabditis elegans; ECANG7: Echinococcus canadensis; Egra: Echinococcus granulosus; Emul: Echinococcus multilocularis; Gsal: Gyrodactylus salaris; Hmic: Hymenolepis microstoma; Sman: Schistosoma mansoni; Smed: Schimdtea mediterranea; TsM1: Taenia solium. (D) Venn diagram of shared loci between the different Echinococcus species. (i) The number of total SNPs in each species using $E$. canadensis (G7) genome as reference. (ii) The number of total SNPs in each species using E. multilocularis genome as reference. (iii) The number of total SNPs in each species using E. granulosus (G1) genome as reference. The numbers under each species name indicate the number of SNPs in the species against the corresponding reference genome. The numbers in the overlap region indicate the number of SNPS with the same polymorphism at the same locus between the species. (PDF $530 \mathrm{~kb}$ )

\section{Abbreviations}

AgB: Taeniidae antigen; CGls: CpG Islands; COX1: Cytochrome oxidase 1; CpG: Pair of adjacent CG; DNMT: DNA methyltransferases; ERAD: Endoplasmic reticulum (ER)-associated degradation; GO: Gene Ontology; GPCR: Adhesion G protein-coupled receptors; GPS: GPCR-proteolytic site; Hsp: Heat shock protein; LINE: Long Interspersed Nuclear Elements; LTR: Long terminal repeat; MBD: Methyl binding domain protein; MeCP: Methyl-CpG binding domain proteins; miRNAs: microRNAs; ORF: Open reading frame; PCR: Polymerase chain reaction; piRNAs: piwiRNAs; RISC: RNA-induced silencing complex; SAM: S-adenosylmethionine; SINE: Short Interspersed Nuclear Elements; siRNAs: Small interfering RNAs; SNP: Single nucleotide polymorphism; SNV: Single nucleotide variation; TRIM: Terminal-repeat retrotransposon in miniature; V-ATPase: Vacuolar ATPase

\section{Acknowledgements}

The authors thank to Uriel Koziol and Klaus Brehm (Universität Würzburg) for kindly providing E. multilocularis genomic DNA and to Dario Carballido for the server administration and maintenance.

\section{Funding}

This study was supported by the National Agency for Science and Technology Promotion (ANPCyT), Argentina, projects PICT 2012 No.2121 (M. R.) and PICT-CABBIO 2012 No. 3044 (L. K.); by the National Scientific and Technical Research Council (CONICET), project PIP114; and by a joint funding between Argentina and Brazil, CAPGBA-CAPES 070/30. Instituto de Investigaciones en Microbiología y Parasitología Médica (IMPaM) and Sistema Nacional 
de Computación de Alto Desempeño (SNCAD) of Ministerio de Ciencia, Tecnología e Innovación Productiva (MINCyT). L. M. and N. M are recipients of the National Scientific and Technical Research Council (CONICET), Argentina, doctoral fellowship. G. O. is a CNPq fellow (309312/2012-4). G. O. received funding from CAPES (REDE 21/2015), CNPq (304138/2014-2) and FAPEMIG (RED-00014-14). Fundación Bunge y Born, Grant for Research on Infectious Diseases 2015/16.

\section{Availability of data and materials}

The datasets generated and/or analysed during the current study are available in http://parasite.wormbase.org/Echinococcus_canadensis_prjeb8 992/Info/Index and https://www.ebi.ac.uk/ena/data/view/PRJEB8992. All data generated or analysed during this study are included in this published article in supplementary material.

\section{Authors' contributions}

LLM genome assembly, genome analysis, CEGMA analysis, gene prediction, gene annotation, OtrhoMCL pipeline, SNPs and comparative genome analyses, database queries, 3D modelling, data interpretation and drafting of the paper; JA, FMGA and ACMS library construction and KEEG analysis; NM, $\mathrm{MC}$ and FC drug target analysis and data interpretation; AF small RNA pathway analysis; LLM, MR, GO and LK design and concept of the study, and writing of the final manuscript. All of the authors read and approved the final manuscript.

\section{Competing interests}

The authors declare that they have no competing interests.

\section{Consent for publication}

Not applicable.

\section{Ethics approval and consent to participate}

Not applicable.

\section{Author details}

${ }^{1}$ IMPaM, CONICET, Facultad de Medicina, Universidad de Buenos Aires, Ciudad Autónoma de Buenos Aires, Argentina. ${ }^{2}$ Genomics and Computational Biology Group, René Rachou Research Center, Oswaldo Cruz Foundation, Belo Horizonte, Brazil. ${ }^{3}$ Instituto Tecnológico Vale, Belém, Brazil.

Received: 21 May 2016 Accepted: 9 February 2017

\section{Published online: 27 February 2017}

\section{References}

1. Sajid M, Rogers J, Rajandream M-A, Berriman M, Haas BJ, LoVerde PT, et al. The genome of the blood fluke Schistosoma mansoni. Nature. 2009;460:3528. Available from: http://www.pubmedcentral.nih.gov/articlerender. fcgi?artid $=2756445 \&$ tool $=$ pmcentrez\&rendertype $=$ abstract.

2. Liu F, Zhou Y, Wang ZQ, Lu G, Zheng H, Brindley et al. The Schistosoma japonicum genome reveals unique features of host-parasite interplay. Nature. 2009;460:345-51.

3. Young ND, Jex AR, Li B, Liu S, Yang L, Xiong Z, et al. Whole-genome sequence of Schistosoma haematobium. Nat Genet. 2012;44:221-5. Available from: http://www.ncbi.nlm.nih.gov/pubmed/22246508.

4. Wang X, Chen W, Huang Y, Sun J, Men J, Liu H, et al. The draft genome of the carcinogenic human liver fluke Clonorchis sinensis. Genome Biol. 2011; 12:R107.

5. Zheng H, Zhang W, Zhang L, Zhang Z, Li J, Lu G, et al. The genome of the hydatid tapeworm Echinococcus granulosus. Nat Genet. 2013;45:1168-75. Available from: http://dx.doi.org/10.1038/ng.2757.

6. Tsai IJ, Zarowiecki M, Holroyd N, Garciarrubio A, Sanchez-Flores A, Brooks KL, et al. The genomes of four tapeworm species reveal adaptations to parasitism. Nature. 2013;496:57-63. Available from: http:// dx.doi.org/10.1038/nature12031.

7. Bennett HM, Mok HP, Gkrania-Klotsas E, Tsai IJ, Stanley EJ, Antoun NM, et al. The genome of the sparganosis tapeworm Spirometra erinaceieuropaei isolated from the biopsy of a migrating brain lesion. Genome Biol. 2014;15: 510. Available from: http://www.pubmedcentral.nih.gov/articlerender. fcgi?artid=4265353\&tool=pmcentrez\&rendertype=abstract.

8. Yang Y, Clements ACA, Gray DJ, Atkinson J-AM, Williams GM, Barnes TS, et al. Impact of anthropogenic and natural environmental changes on
Echinococcus transmission in Ningxia Hui Autonomous Region, the People's Republic of China [Internet]. Parasit. Vectors. 2012:146. Available from: http:// www.parasitesandvectors.com/content/5/1/146.

9. Craig PS, Budke CM, Schantz PM, Li T, Oiu J, Yang Y, et al. Human Echinococcosis: A Neglected Disease? Trop Med Health. 2007;35:283-92. Available from: http://joi.jlc.jst.go.jp/JST.JSTAGE/tmh/35.283?from=CrossRef.

10. Budke CCM, Deplazes P, Torgerson PPR. Global socioeconomic impact of cystic echinococcosis. Emerg Infect Dis. 2006;12:296-303. Available from: http://wwwnc.cdc.gov/eid/article/12/2/05-0499.htm.

11. Sultana N, Hashim TK, Jan SY, Khan Z, Malik T, Shah W. Primary cervical hydatid cyst: a rare occurrence. Diagn Pathol. 2012;7:157. Available from: http://www.diagnosticpathology.diagnomx.eu/. Cited 29 Mar 2016.

12. McManus DP. Current status of the genetics and molecular taxonomy of Echinococcus species. Parasitology. 2013;140:1617-23. Available from: http:// www.ncbi.nlm.nih.gov/pubmed/23750777.

13. Nakao M, Yanagida T, Okamoto M, Knapp J, Nkouawa A, Sako Y, et al. Stateof-the-art Echinococcus and Taenia: Phylogenetic taxonomy of humanpathogenic tapeworms and its application to molecular diagnosis. Infect Genet Evol. 2010;10:444-52.

14. Alvarez Rojas CA, Romig T, Lightowlers MW. Echinococcus granulosus sensu lato genotypes infecting humans-review of current knowledge. Int J Parasitol. 2014;44:9-18. Available from: http://www.ncbi.nlm.nih.gov/pubmed/24269720.

15. Cucher MA, Macchiaroli N, Baldi G, Camicia F, Prada L, Maldonado L, et al. Cystic echinococcosis in South America: Systematic review of species and genotypes of Echinococcus granulosus sensu lato in humans and natural domestic hosts. Trop Med Int Heal. 2015;21:166-75.

16. Schneider R, Gollackner B, Schindl M, Tucek G, Auer H. Echinococcus canadensis $\mathrm{G} 7$ (pig strain): an underestimated cause of cystic echinococcosis in Austria. Am J Trop Med Hyg. 2010;82:871-4. Available from: http://www.pubmedcentral.nih.gov/articlerender.fcgi?artid= 2861383\&tool=pmcentrez\&rendertype=abstract.

17. Sadjjadi SM, Mikaeili F, Karamian M, Maraghi S, Sadjjadi FS, ShariatTorbaghan S, et al. Evidence that the Echinococcus granulosus G6 genotype has an affinity for the brain in humans. Int J Parasitol. 2013;43:875-7. Available from: http://www.ncbi.n/m.nih.gov/pubmed/23891711.

18. Rosenzvit MC, Canova SG, Kamenetzky L, Ledesma BA, Guarnera EA. Echinococcus granulosus: cloning and characterization of a tandemly repeated DNA element. Exp Parasitol. 1997;87:65-8. Available from: http:// www.ncbi.nlm.nih.gov/pubmed/9287959.

19. Rosenzvit M, Zhang L-H, Kamenetzky L, Canova S, Guarnera E, McManus D. Genetic variation and epidemiology of Echinococcus granulosus in Argentina. Parasitology. 1999;118:523-30.

20. Kamenetzky L, Canova SG, Guarnera EA, Rosenzvit MC. Echinococcus granulosus: DNA extraction from germinal layers allows strain determination in fertile and nonfertile hydatid cysts. Exp Parasitol. 2000;95:122-7. Available from: http://www.ncbi.nlm.nih.gov/pubmed/10910713.

21. Kamenetzky L, Muzulin PM, Gutierrez AM, Angel SO, Zaha A, Guarnera EA, et al. High polymorphism in genes encoding antigen $B$ from human infecting strains of Echinococcus granulosus. Parasitology. 2005;131:805-15.

22. Muzulin PM, Kamenetzky L, Gutierrez AM, Guarnera EA, Rosenzvit MC. Echinococcus granulosus antigen B gene family: Further studies of strain polymorphism at the genomic and transcriptional levels. Exp Parasitol. 2008;118:156-64.

23. Eckert J, Thompson RCA, Lymbery AJ, Pawlowski ZS, Gottstein B, Morgan UM. Further evidence for the occurrence of a distinct strain of Echinococcus granulosus in European pigs. Parasitol Res. 1993;79:42-8.

24. Cucher M, Mourglia-Ettlin G, Prada L, Costa H, Kamenetzky L, Poncini C, et al. Echinococcus granulosus pig strain (G7 genotype) protoscoleces did not develop secondary hydatid cysts in mice. Vet Parasitol. 2013;193:185-92.

25. Schantz PM, Van den Bossche H, Eckert J. Chemotherapy for larval echinococcosis in animals and humans: report of a workshop. Zeitschrift für Parasitenkd. 1982;67:5-26. Available from: http://www.ncbi.nlm.nih.gov/ pubmed/7041454.

26. Hemphill A, Stadelmann B, Rufener R, Spiliotis M, Boubaker G, Müller J, et al. Treatment of echinococcosis: albendazole and mebendazole-what else? Parasite. 2014;21. Available from: http://www.ncbi.nlm.nih.gov/pubmed/25526545.

27. Bankevich A, Nurk S, Antipov D, Gurevich AA, Dvorkin M, Kulikov AS, et al. SPAdes: A New Genome Assembly Algorithm and Its Applications to SingleCell Sequencing. J Comput Biol. 2012;19:455-77.

28. Parra G, Bradnam K, Korf I. CEGMA: a pipeline to accurately annotate core genes in eukaryotic genomes. Bioinformatics. 2007;23:1061-7. Available from: http://www.ncbi.nlm.nih.gov/pubmed/17332020. 
29. Holt C, Yandell M. MAKER2: an annotation pipeline and genome-database management tool for second-generation genome projects. BMC Bioinformatics. 2011;12:491. Available from: http://www.pubmedcentral.nih. gov/articlerender.fcgi?artid=3280279\&tool=pmcentrez\&rendertype=abstract.

30. Hahn C, Fromm B, Bachmann L. Comparative genomics of flatworms (Platyhelminthes) reveals shared genomic features of ecto- and endoparastic neodermata. Genome Biol Evol. 2014;6:1105-17.

31. Macchiaroli N, Cucher M, Zarowiecki M, Maldonado L, Kamenetzky L, Rosenzvit MC. microRNA profiling in the zoonotic parasite Echinococcus canadensis using a high-throughput approach. Parasit Vectors. 2015;8:83. Available from: http://www.pubmedcentral.nih.gov/articlerender.fcgi?artid= 4326209\&tool=pmcentrez\&rendertype=abstract.

32. Bermudez-Santana C, Attolini C, Kirsten T, Engelhardt J, Prohaska SJ, Steigele S, et al. Genomic organization of eukaryotic tRNAs. BMC Genomics. 2010;11: 270. Available from: http://bmcgenomics.biomedcentral.com/articles/10. 1186/1471-2164-11-270.

33. Smit Arian HR. RepeatModeler - 1.0.8 [Internet]. Institute for Systems Biology. 2008. Available from: http://www.repeatmasker.org/RepeatModeler.html.

34. Maillard S, Gottstein B, Haag KL, Ma S, Colovic I, Benchikh-Elfegoun MC, et al. The EmsB tandemly repeated multilocus microsatellite: a new tool to investigate genetic diversity of Echinococcus granulosus sensu lato. J Clin Microbiol. 2009;47:3608-16. Available from: http://www.ncbi.nlm.nih.gov/ pubmed/19741078.

35. Bart JM, Abdukader M, Zhang YL, Lin RY, Wang YH, Nakao M, et al. Genotyping of human cystic echinococcosis in Xinjiang, PR China. Parasitology. 2006;133: 571-9. Available from: http://www.ncbi.nlm.nih.gov/pubmed/16836793.

36. Marín M, Garat B, Pettersson U, Ehrlich R. Isolation and characterization of a middle repetitive DNA element from Echinococcus granulosus. Mol Biochem Parasitol. 1993;59:335-8. Available from: http://www.ncbi.nlm.nih.gov/ pubmed/8341331.

37. Koziol U, Radio S, Smircich P, Zarowiecki M, Fernández C, Brehm K. A Novel Terminal-Repeat Retrotransposon in Miniature (TRIM) Is Massively Expressed in Echinococcus multilocularis Stem Cells. Genome Biol Evol. 2015;7:2136-53. Available from: http://www.pubmedcentral.nih.gov/articlerender.fcgi?artid= 4558846\&tool=pmcentrez\&rendertype=abstract.

38. Bogdanović O, Veenstra GJC. DNA methylation and methyl-CpG binding proteins: developmental requirements and function. Chromosoma. 2009;118: 549-65. Available from: http://www.ncbi.nlm.nih.gov/pubmed/19506892.

39. Klose RJ, Bird AP. Genomic DNA methylation: the mark and its mediators. Trends Biochem Sci. 2006;31:89-97. Available from: http://www.ncbi.nlm.nih. gov/pubmed/16403636.

40. Geyer KK, Rodriguez Lopez CM, Chalmers IW, Munshi SE, Truscott M, Heald J, et al. Cytosine methylation regulates oviposition in the pathogenic blood fluke Schistosoma mansoni. Nat Commun. 2011;2:424. Available from: http:// www.ncbi.nlm.nih.gov/pubmed/21829186.

41. Geyer KK, Chalmers IW, Mackintosh N, Hirst JE, Geoghegan R, Badets M, et al. Cytosine methylation is a conserved epigenetic feature found throughout the phylum Platyhelminthes. BMC Genomics. 2013;14:462. Available from: http://www.pubmedcentral.nih.gov/articlerender.fcgi?artid= 3710501\&tool=pmcentrez\&rendertype=abstract.

42. Hendrich B, Tweedie $\mathrm{S}$. The methyl-CpG binding domain and the evolving role of DNA methylation in animals. Trends Genet. 2003;19:269-77. Available from: http://www.ncbi.n/m.nih.gov/pubmed/12711219.

43. Moore LD, Le T, Fan G. DNA methylation and its basic function. Neuropsychopharmacology [Internet]. Nat Publ Group. 2013;38:23-38. Available from: http://www.pubmedcentral.nih.gov/articlerender.fcgi?artid= 3521964\&tool=pmcentrez\&rendertype=abstract.

44. Saxonov S, Berg P, Brutlag DL. A genome-wide analysis of CpG dinucleotides in the human genome distinguishes two distinct classes of promoters. Proc Natl Acad Sci U S A. 2006;103:1412-7. Available from: http://www.ncbi.nlm.nih.gov/pubmed/16432200

45. Medvedeva YA, Fridman MV, Oparina NJ, Malko DB, Ermakova EO, Kulakovskiy IV, et al. Intergenic, gene terminal, and intragenic CpG islands in the human genome. BMC Genomics. 2010;11:48. Available from: http:// www.ncbi.nlm.nih.gov/pubmed/20085634.

46. Carninci P, Sandelin A, Lenhard B, Katayama S, Shimokawa K, Ponjavic J, et al. Genome-wide analysis of mammalian promoter architecture and evolution. Nat Genet. 2006;38:626-35. Available from: http://www.ncbi.nlm. nih.gov/pubmed/16645617.

47. Mohn F, Weber M, Rebhan M, Roloff TC, Richter J, Stadler MB, et al. Lineagespecific polycomb targets and de novo DNA methylation define restriction and potential of neuronal progenitors. Mol Cell. 2008;30:755-66. Available from: http://www.ncbi.nlm.nih.gov/pubmed/18514006.

48. Han L, Su B, Li W-H, Zhao Z. CpG island density and its correlations with genomic features in mammalian genomes. Genome Biol. 2008;9:R79. Available from: http://genomebiology.com/2008/9/5/R79.

49. Cucher M, Prada L, Mourglia-Ettlin G, Dematteis S, Camicia F, Asurmendi S, et al. Identification of Echinococcus granulosus microRNAs and their expression in different life cycle stages and parasite genotypes. Int J Parasitol. 2011;41:43948. Available from: http://www.ncbi.nlm.nih.gov/pubmed/21219906.

50. Mara Rosenzvit, Marcela Cucher, Laura Kamenetzky, Natalia Macchiaroli, Laura Prada FC. MicroRNAs in Endoparasites. In: James C. Johnson, editor. MicroRNA Non-Coding RNA Technol. Dev. Appl. [Internet]. Buenos Aires; 2013. p. 65-92. Available from: https://www.novapublishers.com/catalog/ product_info.php?products_id=37973.

51. Elkayam E, Kuhn C-D, Tocilj A, Haase AD, Greene EM, Hannon GJ, et al. The structure of human argonaute-2 in complex with miR-20a. Cell. 2012;150: 100-10. Available from: http://www.ncbi.n/m.nih.gov/pubmed/22682761.

52. Schürmann N, Trabuco LG, Bender C, Russell RB, Grimm D. Molecular dissection of human Argonaute proteins by DNA shuffling. Nat Struct Mol Biol. 2013;20:818-26. Available from: http://www.ncbi.nlm.nih.gov/ pubmed/23748378.

53. Nakao M, Yanagida T, Konyaev S, Lavikainen A, Odnokurtsev VA, Zaikov VA, et al. Mitochondrial phylogeny of the genus Echinococcus (Cestoda: Taeniidae) with emphasis on relationships among Echinococcus canadensis genotypes. Parasitology. 2013;140:1625-36. Available from: http://www.ncbi. nlm.nih.gov/pubmed/23731519.

54. Pan W, Shen Y, Han X, Wang Y, Liu H, Jiang Y, et al. Transcriptome Profiles of the Protoscoleces of Echinococcus granulosus Reveal that Excretory-Secretory Products Are Essential to Metabolic Adaptation. PLoS Negl Trop Dis. 2014:8:1-15.

55. Hewitson JP, Grainger JR, Maizels RM. Helminth immunoregulation: The role of parasite secreted proteins in modulating host immunity. Mol Biochem Parasitol. 2009;167:1-11.

56. Nono JK, Pletinckx K, Lutz MB, Brehm K. Excretory/secretory-products of Echinococcus multilocularis larvae induce apoptosis and tolerogenic properties in dendritic cells in vitro. PLoS Negl Trop Dis. 2012;6:e1516.

57. Robinson MW, Donnelly S, Hutchinson AT, To J, Taylor NL, Norton RS, et al. A family of helminth molecules that modulate innate cell responses via molecular mimicry of host antimicrobial peptides. PLoS Pathog. 2011;7: e1002042. Available from: http://www.ncbi.nlm.nih.gov/pubmed/21589904.

58. Thivierge K, Cotton S, Schaefer DA, Riggs MW, To J, Lund ME, et al. Cathelicidin-like helminth defence molecules (HDMs): absence of cytotoxic, anti-microbial and anti-protozoan activities imply a specific adaptation to immune modulation. PLoS Negl Trop Dis. 2013;7:e2307. Available from: http://www.ncbi.nlm.nih.gov/pubmed/23875042.

59. Yi S. Birds do it, bees do it, worms and ciliates do it too: DNA methylation from unexpected corners of the tree of life. 2012.

60. Han L, Zhao Z. Contrast features of CpG islands in the promoter and other regions in the dog genome. Genomics. 2009;94:117-24. Available from: http://dx.doi.org/10.1016/j.ygeno.2009.04.007.

61. Jiang C, Han L, Su B, Li W-H, Zhao Z. Features and trend of loss of promoter-associated $\mathrm{CpG}$ islands in the human and mouse genomes. Mol Biol Evol. 2007;24:1991-2000. Available from: http://www.ncbi.nlm.nih.gov/ pubmed/17591602.

62. Matsuo K, Clay O, Takahashi T, Silke J, Schaffner W. Evidence for erosion of mouse CpG islands during mammalian evolution. Somat Cell Mol Genet. 1993; 19:543-55. Available from: http://www.ncbi.nlm.nih.gov/pubmed/8128314.

63. Zhao Z, Jiang C. Methylation-dependent transition rates are dependent on local sequence lengths and genomic regions. Mol Biol Evol. 2007;24:23-5. Available from: http://www.ncbi.nlm.nih.gov/pubmed/17056644.

64. Fryxell KJ, Moon W-J. CpG mutation rates in the human genome are highly dependent on local GC content. Mol Biol Evol. 2005;22:650-8. Available from: http://www.ncbi.nlm.nih.gov/pubmed/15537806.

65. Wasik K, Gurtowski J, Zhou X, Ramos OM, Delás MJ, Battistoni G, et al. Genome and transcriptome of the regeneration-competent flatworm, Macrostomum lignano. Proc Natl Acad Sci. 2015;112:201516718. Available from: http://www.pnas.org/content/112/40/12462.abstract.

66. Saarma U, Jõgisalu I, Moks E, Varcasia A, Lavikainen A, Oksanen A, et al. A novel phylogeny for the genus Echinococcus, based on nuclear data, challenges relationships based on mitochondrial evidence. Parasitology. 2009;136:317-28.

67. Kamenetzky L, Gutierrez AM, Canova SG, Haag KL, Guarnera EA, Parra A, et al. Several strains of Echinococcus granulosus infect livestock and humans in 
Argentina. Infect Genet Evol. 2002;2:129-36. Available from: http://www. ncbi.nlm.nih.gov/pubmed/12797989.

68. Bolger AM, Lohse M, Usadel B. Trimmomatic: a flexible trimmer for Illumina sequence data. Bioinformatics. 2014;30:2114-20. Available from: http://www. ncbi.nlm.nih.gov/pubmed/24695404.

69. Zerbino DR, Birney E. Velvet: Algorithms for de novo short read assembly using de Bruijn graphs. Genome Res. 2008;18:821-9.

70. Luo R, Liu B, Xie Y, Li Z, Huang W, Yuan J, et al. SOAPdenovo2: an empirically improved memory-efficient short-read de novo assembler. Gigascience. 2012;1:18. Available from: http://www.gigasciencejournal.com/ content/1/1/18\%5Cn and http://www.pubmedcentral.nih.gov/articlerender. fcgi?artid $=3626529 \&$ tool $=$ pmcentrez\&rendertype $=$ abstract.

71. Safonova Y, Bankevich A, Pevzner PA. dipSPAdes: Assembler for Highly Polymorphic Diploid Genomes. J Comput Biol. 2015;22:528-45. Available from: http://www.ncbi.nlm.nih.gov/pubmed/25734602.

72. Tsai IJ, Otto TD, Berriman M. Improving draft assemblies by iterative mapping and assembly of short reads to eliminate gaps. Genome Biol. 2010;11:R41. Available from: http://www.pubmedcentral.nih.gov/ articlerender.fcgi?artid=2884544\&tool=pmcentrez\&rendertype=abstract.

73. Otto TD, Sanders M, Berriman M, Newbold C. Iterative correction of reference Nucleotides (iCORN) using second generation sequencing technology. Bioinformatics. 2010;26:1704-7.

74. Assefa S, Keane TM, Otto TD, Newbold C, Berriman M. ABACAS: algorithmbased automatic contiguation of assembled sequences. Bioinformatics. 2009;25:1968-9. Available from: http://www.pubmedcentral.nih.gov/ articlerender.fcgi?artid=2712343\&tool=pmcentrez\&rendertype=abstract.

75. Gurevich A, Saveliev V, Vyahhi N, Tesler G. QUAST: Quality assessment tool for genome assemblies. Bioinformatics. 2013;29:1072-5.

76. Stanke M, Waack S. Gene prediction with a hidden Markov model and a new intron submodel. Bioinformatics. 2003;19 Suppl 2:ii215-25. Available from: http://www.ncbi.nlm.nih.gov/pubmed/14534192.

77. Korf I. Gene finding in novel genomes. BMC Bioinformatics. 2004:5:59

78. Slater GSC, Birney E. Automated generation of heuristics for biological sequence comparison. BMC Bioinformatics. 2005;6:31. Available from: http:// www.ncbi.nlm.nih.gov/pubmed/15713233.

79. Kim D, Pertea G, Trapnell C, Pimentel H, Kelley R, Salzberg SL. TopHat2: accurate alignment of transcriptomes in the presence of insertions, deletions and gene fusions. Genome Biol. 2013;14:R36. Available from: http://genomebiology.com/2013/14/4/R36.

80. Trapnell C, Pachter L, Salzberg SL. TopHat: Discovering splice junctions with RNA-Seq. Bioinformatics. 2009;25:1105-11.

81. Lomsadze A, Ter-Hovhannisyan V, Chernoff YO, Borodovsky M. Gene identification in novel eukaryotic genomes by self-training algorithm. Nucleic Acids Res. 2005;33:6494-506. Available from: http://www.ncbi.nlm. nih.gov/pubmed/16314312.

82. Lomsadze A, Burns PD, Borodovsky M. Integration of mapped RNA-Seq reads into automatic training of eukaryotic gene finding algorithm. Nucleic Acids Res. 2014;42:e119. Available from: http://www.pubmedcentral.nih.gov/ articlerender.fcgi?artid $=4150757 \&$ tool $=$ pmcentrez\&rendertype $=$ abstract.

83. Smit AFA, Hubley R GP. RepeatMasker 4.0.6 [Internet]. 2010. Available from: http://www.repeatmasker.org/RMDownload.html.

84. Lowe TM, Eddy SR. tRNAscan-SE: a program for improved detection of transfer RNA genes in genomic sequence. Nucleic Acids Res. 1997;25:955-64.

85. Altschul SF, Madden TL, Schäffer AA, Zhang J, Zhang Z, Miller W, et al. Gapped BLAST and PSI-BLAST: a new generation of protein database search programs. Nucleic Acids Res. 1997;25:3389-402. Available from: http://www. ncbi.nlm.nih.gov/pubmed/9254694.

86. Abrusán G, Grundmann N, DeMester L, Makalowski W. TEclass-a tool for automated classification of unknown eukaryotic transposable elements. Bioinformatics. 2009;25:1329-30. Available from: http://www.ncbi.nlm.nih. gov/pubmed/19349283.

87. Keibler E, Brent MR. Eval: a software package for analysis of genome annotations. BMC Bioinformatics. 2003;4:50. Available from: http:// bmcbioinformatics.biomedcentral.com/articles/10.1186/1471-2105-4-50.

88. Quevillon E, Silventoinen V, Pillai S, Harte N, Mulder N, Apweiler R, et al. InterProScan: protein domains identifier. Nucleic Acids Res. 2005;33:W1 16-20. Available from: http://www.ncbi.nlm.nih.gov/pubmed/15980438.

89. Ashburner M, Ball CA, Blake JA, Botstein D, Butler H, Cherry JM, et al. Gene ontology: tool for the unification of biology. The Gene Ontology Consortium. Nat Genet. 2000;25:25-9. Available from: http://www.ncbi.nlm. nih.gov/pubmed/10802651.
90. Li L, Stoeckert CJJ, Roos DS. OrthoMCL: Identification of Ortholog Groups for Eukaryotic Genomes. Genome Res. 2003;13:2178-89. Available from: http:// genome.cshlp.org/cgi/content/full/13/9/2178.

91. Fischer S, Brunk BP, Chen F, Gao X, Harb OS, lodice JB, et al. Using OrthoMCL to assign proteins to OrthoMCL-DB groups or to cluster proteomes into new ortholog groups. Curr. Protoc. Bioinformatics [Internet]. 2011;Chapter 6:Unit 6.12.1-19. Available from: http://www.ncbinlm.nih.gov/ pubmed/21901743. Cited 31 Mar 2016.

92. Finn RD, Mistry J, Schuster-Böckler B, Griffiths-Jones S, Hollich V, Lassmann T, et al. Pfam: clans, web tools and services. Nucleic Acids Res. 2006;34:D24751. Available from: http://www.ncbi.nlm.nih.gov/pubmed/16381856.

93. Kelley LA, Mezulis S, Yates CM, Wass MN, Sternberg MJE. The Phyre2 web portal for protein modeling, prediction and analysis. Nat Protoc. 2015;10: 845-58. Available from: http://www.ncbi.nlm.nih.gov/pubmed/25950237.

94. Biasini M, Bienert S, Waterhouse A, Arnold K, Studer G, Schmidt T, et al. SWISS-MODEL: modelling protein tertiary and quaternary structure using evolutionary information. Nucleic Acids Res. 2014;42:W252-8. Available from: http://www.ncbi.n/m.nih.gov/pubmed/24782522.

95. Arnold K, Bordoli L, Kopp J, Schwede T. The SWISS-MODEL workspace: a web-based environment for protein structure homology modelling. Bioinformatics. 2006;22:195-201. Available from: http://www.ncbi.nlm.nih. gov/pubmed/16301204.

96. Kiefer F, Arnold K, Künzli M, Bordoli L, Schwede T. The SWISS-MODEL Repository and associated resources. Nucleic Acids Res. 2009;37:D387-92. Available from: http://www.ncbi.nlm.nih.gov/pubmed/18931379.

97. Guex N, Peitsch MC, Schwede T. Automated comparative protein structure modeling with SWISS-MODEL and Swiss-PdbViewer: a historical perspective. Electrophoresis. 2009;30 Suppl 1:S162-73. Available from: http://www.ncbi. nlm.nih.gov/pubmed/19517507.

98. Takai D, Jones PA. Comprehensive analysis of $\mathrm{CpG}$ islands in human chromosomes 21 and 22. Proc Natl Acad Sci U S A. 2002;99:3740-5. Available from: http://www.ncbi.nlm.nih.gov/pubmed/11891299.

99. Krzywinski M, Schein J, Birol I, Connors J, Gascoyne R, Horsman D, et al. Circos: an information aesthetic for comparative genomics. Genome Res. 2009;19: 1639-45. Available from: http://www.ncbi.nlm.nih.gov/pubmed/19541911.

100. Langmead B, Salzberg SL. Fast gapped-read alignment with Bowtie 2. Nat Methods. 2012;9:357-9. Available from: http://www.ncbi.nlm.nih.gov/ pubmed/22388286.

101. Li H, Durbin R. Fast and accurate short read alignment with BurrowsWheeler transform. Bioinformatics. 2009;25:1754-60. Available from: http:// www.pubmedcentral.nih.gov/articlerender.fcgi?artid=2705234\&tool= pmcentrez\&rendertype $=$ abstract.

102. Danecek P, Auton A, Abecasis G, Albers CA, Banks E, DePristo MA, et al. The variant call format and VCFtools. Bioinformatics. 2011;27:2156-8.

103. Cingolani $P$, Platts $A$, Wang $L L$, Coon $M$, Nguyen $T$, Wang $L$, et al. A program for annotating and predicting the effects of single nucleotide polymorphisms, SnpEff: SNPs in the genome of Drosophila melanogaster strain w 1118; iso-2; iso-3. Fly (Austin). 2012;6:80-92.

104. Moriya Y, Itoh M, Okuda S, Yoshizawa AC, Kanehisa M. KAAS: an automatic genome annotation and pathway reconstruction server. Nucleic Acids Res 2007;35:W182-5

105. Otto TD, Dillon GP, Degrave WS, Berriman M. RATT: Rapid Annotation Transfer Tool. Nucleic Acids Res. 2011;39:1-7.

106. Nakao M, Sako Y, Yokoyama N, Fukunaga M, Ito A. Mitochondrial genetic code in cestodes. Mol Biochem Parasitol. 2000;111:415-24. Available from: http://www.ncbi.nlm.nih.gov/pubmed/11163447.

107. Jeanmougin F, Thompson JD, Gouy M, Higgins DG, Gibson TJ. Multiple sequence alignment with Clustal X. Trends Biochem Sci. 1998;23:403-5.

108. Nakao M, McManus DP, Schantz PM, Craig PS, Ito A. A molecular phylogeny of the genus Echinococcus inferred from complete mitochondrial genomes. Parasitology. 2007;134:713-22. Available from: http://www.ncbi.nlm.nih.gov/ pubmed/17156584.

109. Nakao M, Yokoyama N, Sako Y, Fukunaga M, Ito A. The complete mitochondrial DNA sequence of the cestode Echinococcus multilocularis (Cyclophyllidea: Taeniidae). Mitochondrion. 2002;1:497-509. Available from: http://www.ncbi.nlm.nih.gov/pubmed/16120302. 